\title{
Critical behavior of a one-dimensional monomer-dimer reaction model with lateral interactions.
}

\author{
Roberto A. Monetti \\ Institut für Theoretische Physik \\ Physik-Department der Technischen Universität München \\ James-Franck-Strasse, 85747 Garching, Germany
}

21st November 2018

\begin{abstract}
A monomer-dimer reaction lattice model with lateral repulsion among the same species is studied using a mean-field analysis and Monte Carlo simulations. For weak repulsions, the model exhibits a first-order irreversible phase transition between two absorbing states saturated by each different species. Increasing the repulsion, a reactive stationary state appears in addition to the saturated states. The irreversible phase transitions from the reactive phase to any of the saturated states are continuous and belong to the directed percolation universality class. However, a different critical behavior is found at the point where the directed percolation phase boundaries meet. The values of the critical exponents calculated at the bicritical point are in good agreement with the exponents corresponding to the parity-conserving universality class. Since the adsorption-reaction processes does not lead to a non-trivial local parity-conserving dynamics, this result confirms that the twofold symmetry between absorbing states plays a relevant role in determining the universality class. The value of the exponent $\delta_{2}$, which characterizes the fluctuations of an interface at the bicritical point, supports the Bassler-Brown's conjecture which states that this is a new exponent in the parity-conserving universality class.
\end{abstract}




\section{Introduction}

Nonequilibrium models are relevant to a broad scope of phenomena in physics, chemistry, biophysics, ecology, etc. A relevant feature of the nonequilibrium models which exhibit a second-order irreversible phase transition (IPT) to a unique absorbing state is that their critical behavior is in the directed percolation (DP) universality class $\mathbb{1}$. DP critical behavior is observed over wide-ranging problems emerging from different disciplines such as quantum particle physics [2], irreversible catalytic systems [3, [4, 5], the contact process [6], branching annihilating random walks with an odd number of offspring [7], etc. This fact led Janssen and Grassberger to conjecture that in onecomponent models continuous transitions to a single absorbing state are in the DP universality class [8]. Motivated by these findings several models with multiple absorbing states were proposed but no new universality class was found [9]. This proved that a greater number of absorbing states is not enough to obtain a critical behavior different from DP. Thus the DP universality class is apparently extremely robust.

In contrast to the well-established DP universality class, only a few exceptions are known that do not belong to this class. The known examples are models $\mathrm{A}$ and $\mathrm{B}$ of probabilistic cellular automata [10, 11, nonequilibrium kinetic Ising models [12, the interacting monomer-dimer model with infinite repulsion [13], and the branching annihilating random walks with an even number of offsprings [14, 15, 16]. A relevant feature is shared by all these models: the number of particles (or kinks) is conserved modulo 2. That is why this new universality class is sometimes called parity-conserving (PC) class.

Recently, a hierarchy of unidirectionally coupled DP processes has been studied [17]. It has been shown by means of field-theoretic renormalization group techniques and Monte Carlo simulations that new values of the exponent $\beta$ corresponding to the order parameter arise at the multicritical point while the dynamical critical exponents take the same values as the ones corresponding to DP. This new result again poses the question of whether a few distinct universality classes are enough to characterize the critical behavior of nonequilibrium systems [17].

Few models that display a phase transition in the PC class where no explicit conservation law is present have appeared in the literature. The known examples are the generalized Domany-Kinzel cellular automata [18], the three-species monomer-monomer 
model [19] and the monomer-monomer surface reaction model [20, 21]. Since these models do not explicitly conserve any quantity modulo 2 they show that rather than parity conservation the symmetry among absorbing states is the origin for the emergence of a different class [18, 22].

In this work we study the behavior of the one-dimensional monomer-dimer surface reaction model with lateral repulsion by means of a mean-field (MF) analysis and Monte Carlo simulations. This model is an extension in one dimension of the well-known Ziff, Gulari, and Barshad surface-reaction model [3]. The model was first proposed by Kim and Park [13] and studied in the case of infinitely strong repulsions. For finite repulsions the model has a rich critical behavior displaying first- and second-order IPT.

The manuscript is organized as follows. In section II we begin with a brief description of the model and show the phase diagram obtained by simulations. In section III we present the MF analysis and compare MF results with simulation results. Section IV contains a detailed analysis of the critical behavior of the model performed by means of Monte Carlo simulations. In the last section we summarize our results.

\section{The Model}

The model we study in this paper was first introduced by Kim and Park [13]. The monomer-dimer reaction model with lateral interactions can be defined as follows: A monomer $A$ or a dimer $B_{2}$ adsorb at the vacant sites of a one-dimensional lattice with probabilities $p$ and $q$ respectively, where $p+q=1$. Each adsorption attempt begins by selecting one site of the lattice at random and if that site is occupied the trial ends. Otherwise, if the site is empty, an $A\left(B_{2}\right)$ species is selected with probability $p$ $(q)$, respectively. In order to introduce lateral interactions between the same kind of particles, it is assumed that the adsorption probability of the selected species depends on the configuration of the adsorbed particles on the nearest neighboring (NN) sites of the selected one. Then the adsorption probability $P_{A}$ can be written as

$$
P_{A}= \begin{cases}p & \text { if } \nexists \text { NN A } \\ p\left(1-r_{A}\right) & \text { if } \exists \text { NN A },\end{cases}
$$

where $0 \leq r_{A}<1$. For the adsorption of $B_{2}$ one has first to select at random a NN site of the empty one and if that site is occupied the trial ends because the dimer can not be deposited on the lattice. Otherwise, if the site is empty, the adsorption probability 
$P_{B_{2}}$ is given by

$$
P_{B_{2}}= \begin{cases}q & \text { if } \nexists \text { NN B } \\ q\left(1-r_{B}\right) & \text { if } \exists \text { NN B. }\end{cases}
$$

where $0 \leq r_{B}<1$. In this way, the parameters $r_{A}$ and $r_{B}$ can be interpreted as the repulsive interactions among similar species. Unlike species on adjacent sites react immediately and leave the lattice, leading to a process limited only by adsorption.

In this work we study the case $r_{A}=r_{B}=r$. Previous studies of this model [13, 22] focused on various aspects of the critical behavior for infinite repulsive lateral interactions, i. e. $r_{A}=r_{B}=1$. In this case, there are two equivalent absorbing states whose configurations are given only by monomers occupying the odd or even-numbered lattice sites respectively. The critical behavior of this model was found to be in the PC universality class.

Figure 11 shows the phase diagram of the system obtained by both static and dynamical Monte Carlo simulations. There are two different absorbing states characterized by the lattice saturated by $A$ or $B$ species respectively. For weak repulsions the system displays first order IPT between the saturated states, and no reactive phase is observed. The first order critical points have been calculated by means of static Monte Carlo simulations since correlations at first order IPT are short ranged. Increasing the repulsion a reactive window appears whose edges are second order critical lines that separate this state from the absorbing states. The second order IPT have been calculated using dynamic Monte Carlo simulations since fluctuations in this case are imposing.

For $r=1$ (infinite repulsion) [13] one can identify since the very beginning the presence of two equivalent absorbing states. Although one can not find a priori a situation like this for $0 \leq r<1$, a phase point where both absorbing states are statistically equivalent can exist. This happens exactly where both second order lines meet, i. e. at the bicritical point. A similar phase diagram was also found in the interacting monomer-monomer reaction model [20]. Very recently, a careful study at the bicritical point led to the conclusion that this point is in the PC universality class [21]. Given the symmetry of the adsorption process in the interacting monomer-monomer reaction model the bicritical point was found on the line $p=q=0.5$. Since the model we study in this work does not display this symmetry, the localization of the bicritical point is more difficult. 


\section{Mean-Field Theory}

In order to obtain a qualitative understanding of the behavior of the model a mean-field analysis is performed. We consider mean-field approximations [23] that study the time evolution of blocks up to three sites, neglecting higher-order correlations.

We start considering a one-dimensional system of size $L$ at time $t$. Each site can be only at three different states, namely, $A, B$, or $V$ corresponding to a site occupied by a particle of type $A, B$ or empty, respectively. In order to calculate MF approximations taking into account correlations up to three lattice sites, we write down the following MF rate equations

$$
\begin{aligned}
\frac{d \rho_{A}}{d t}= & p \rho_{V V V}+p(1-r)\left(\rho_{A V A}+\rho_{A V V}+\rho_{V V A}\right)-q(1-r)\left(\rho_{B V V A}+\rho_{A V V B}\right) \\
& -q\left(2 \rho_{A V V A}+\rho_{A V V V}+\rho_{V V V A}\right),
\end{aligned}
$$

$$
\begin{gathered}
\frac{d \rho_{B}}{d t}=q\left(2 \rho_{V V V V}+\rho_{A V V V}+\rho_{V V V A}\right)+q(1-r)\left(\rho_{B V V V}+\rho_{V V V B}+\rho_{B V V}+\rho_{V V B}\right) \\
-p(1-r)\left(\rho_{A V B}+\rho_{B V A}\right)-p\left(\rho_{B V B}+\rho_{B V V}+\rho_{V V B}\right) \\
\frac{d \rho_{A A}}{d t}=p(1-r)\left(\rho_{V V A}+\rho_{A V V}+2 \rho_{A V A}\right)-q(1-r)\left(\rho_{A A V V B}+\rho_{B V V A A}\right) \\
-q\left(\rho_{A A V V A}+\rho_{A V V A A}+\rho_{A A V V V}+\rho_{V V V A A}\right)
\end{gathered}
$$

$$
\begin{aligned}
\frac{d \rho_{B B}}{d t}= & q \rho_{V V V V}-p(1-r)\left(\rho_{B B V A}+\rho_{A V B B}\right) \\
& +q(1-r)\left(\rho_{B V V B}+\rho_{B V V V}+\rho_{V V V B}+\rho_{B V V}+\rho_{V V B}\right) \\
& -p\left(\rho_{V V B B}+\rho_{B B V V}+\frac{1}{2} \rho_{B B V B}+\frac{1}{2} \rho_{B V B B}\right),
\end{aligned}
$$

$$
\begin{aligned}
\frac{d \rho_{A A A}}{d t}= & p(1-r)\left(\rho_{V V A A}+\rho_{A A V V}+\rho_{A V A}+\rho_{A A V A}+\rho_{A V A A}\right) \\
& -q(1-r)\left(\rho_{B V V A A A}+\rho_{A A A V V B}\right) \\
& -q\left(\rho_{A A A V V A}+\rho_{A V V A A A}+\rho_{V V V A A A}+\rho_{A A A V V V}\right),
\end{aligned}
$$




$$
\begin{aligned}
\frac{d \rho_{B B B}}{d t}= & q(1-r)\left(2 \rho_{B V V B}+\rho_{V V B B}+\rho_{B B V V}+\rho_{B V V V}+\rho_{V V V B}\right) \\
& -p(1-r)\left(\rho_{B B B V A}+\rho_{A V B B B}\right) \\
& -p\left(\rho_{V V B B B}+\rho_{B B B V V}+\frac{1}{2} \rho_{B V B B B}+\frac{1}{2} \rho_{B B B V B}\right) \\
\frac{d \rho_{A V A}}{d t}= & p\left(\rho_{A V V V}+\rho_{V V V A}\right)+2 p(1-r) \rho_{A V V A}-p(1-r) \rho_{A V A} \\
& -q(1-r)\left(\rho_{B V V A V A}+\rho_{A V A V V B}\right) \\
& -q\left(\rho_{A V A V V A}+\rho_{A V V A V A}+\rho_{V V V A V A}+\rho_{A V A V V V}\right) \\
\frac{d \rho_{B V B}}{d t}= & q\left(\rho_{B V V V V}+\rho_{V V V V B}+\rho_{A V V V B}+\rho_{B V V V A}\right) \\
& +2 q(1-r) \rho_{B V V V B}-p(1-r)\left(\rho_{B V B V A}+\rho_{A V B V B}\right) \\
& -p\left(\rho_{V V B V B}+\rho_{B V B V V}+\rho_{B V B V B}+\rho_{B V B}\right)
\end{aligned}
$$

and

$$
\begin{aligned}
\frac{d \rho_{A V B}}{d t}= & p \rho_{V V V B}+q\left(\rho_{V V V V A}+\rho_{A V V V A}\right)+p(1-r) \rho_{A V V B} \\
& +q(1-r) \rho_{A V V V B}-p\left(\rho_{A V B V V}+\frac{1}{2} \rho_{A V B V B}\right)-p(1-r) \rho_{A V B V A} \\
& -q\left(\rho_{V V V A V B}+\rho_{A V V A V B}\right)-q(1-r) \rho_{B V V A V B}-p(1-r) \rho_{A V B}
\end{aligned}
$$

where $\rho_{i_{1} i_{2} i_{3} \ldots}$ is the density of the block $i_{1} i_{2} i_{3} \ldots$ and we have used the equations that link the density of an $n$-block and the density of an $n+1$-block

$$
\rho_{i_{1} i_{2} \ldots i_{n}}=\sum_{i_{n+1}} \rho_{i_{1} i_{2} \ldots i_{n} i_{n+1}}=\sum_{i_{0}} \rho_{i_{0} i_{1} i_{2} \ldots i_{n}}
$$

where $A B$ pairs are not allowed since they immediately reacts. The processes considered to obtain equations (3) to (11) are listed in table 1. Furthermore, we obviously have the following constraint

$$
\rho_{A}+\rho_{B}+\rho_{V}=1
$$




\begin{tabular}{||c||c||c||c||}
\hline \multicolumn{4}{|c|}{ Table 1: Probabilities for the allowed kinetic processes. } \\
\hline \hline$A$ adsorption & Rate & $B_{2}$ adsorption & Rate \\
\hline$V V V \rightarrow V A V$ & $p$ & $V V V V \rightarrow V B B V$ & $q$ \\
\hline$V V A \rightarrow V A A$ & $p(1-r)$ & $B V V V \rightarrow B B B V$ & $q(1-r)$ \\
\hline$A V V \rightarrow A A V$ & $p(1-r)$ & $V V V B \rightarrow V B B B$ & $q(1-r)$ \\
\hline$A V A \rightarrow A A A$ & $p(1-r)$ & $B V V B \rightarrow B B B B$ & $q(1-r)$ \\
\hline$V V B \rightarrow V V V$ & $p$ & $A V V V \rightarrow V V B V$ & $q$ \\
\hline$B V V \rightarrow V V V$ & $p$ & $V V V A \rightarrow V B V V$ & $q$ \\
\hline$B V B \rightarrow B V V$ & $1 / 2 p$ & $A V V A \rightarrow V V V V$ & $q$ \\
\hline$B V B \rightarrow V V B$ & $1 / 2 p$ & - & - \\
\hline$A V B \rightarrow A V V$ & $p(1-r)$ & $A V V B \rightarrow V V B B$ & $q(1-r)$ \\
\hline$B V A \rightarrow V V A$ & $p(1-r)$ & $B V V A \rightarrow B B V V$ & $q(1-r)$ \\
\hline
\end{tabular}

In the simple MF analysis we just neglect correlations among sites, i. e. $\rho_{i_{1} i_{2} i_{3} \ldots i_{m}} \approx$ $\rho_{i_{1}} \rho_{i_{2}} \rho_{i_{3}} \ldots \rho_{i_{m}}$. Within this approximation, equations (3), (田), and (13), comprise a close set of equations. Then a solution for $\rho_{A}, \rho_{B}$, and $\rho_{V}$ can be obtained. However, more equations are needed in order to obtain results for higher order MF analysis. In the pair MF approach we approximate the density of blocks longer than two sites in the following way

$$
\rho_{i_{1} i_{2} i_{3} \ldots i_{m}} \approx \rho_{i_{1} i_{2}} \prod_{j=2}^{m-1} \frac{\rho_{i_{j} i_{j+1}}}{\rho_{i_{j}}}
$$

Using equations (3)-(6), (13) and writing down the equations (12) which relate singlesite densities with pair densities a solution in the pair-MF approximation can be obtained. Proceeding in a similar way, within the three-sites MF approximation density of blocks longer than three sites are replaced by

$$
\rho_{i_{1} i_{2} i_{3} \ldots i_{m}} \approx \rho_{i_{1} i_{2} i_{3}} \prod_{j=2}^{m-2} \frac{\rho_{i_{j} i_{j+1} i_{j+2}}}{\rho_{i_{j} i_{j+1}}}
$$

Due to the immediate reaction of $A B$ pairs, the stationary densities of triples $A V B$ and $B V A$ are equal. Then, by means of equations (3) to (11), and (13), and considering all the relations between single-site densities, density of pairs and density of triples, the three-sites MF approximation can be solved. It should be pointed out that due to the complexity of the set of equations all the MF approximations have been solved numerically.

Figure 2 shows plots of the densities of $A$ and $B$ in the stationary state versus $p$ and $q$ respectively for two different values of the repulsion $r$ obtained by static Monte Carlo simulations, simple, pair, and three-sites MF analysis. For weak repulsion $(r=0.5)$ 
the sharp jump observed in both the densities of $A$ and $B$ is the signature of first order IPT. Since correlations at a first order IPT are finite we expect the MF approaches to give good results. Although the simple MF approximation is quite poor, results quickly improve for the pair and three-sites approaches. For higher values of the repulsion $r$ the sharp variation in the densities is no longer present but a smooth transition is observed. In general, MF theories do not give good results near second order continuous IPT since second order IPT are governed by fluctuations. However, as it is observed in figures 2c and $2 \mathrm{~d}$, we still obtain a fairly good agreement between the three-sites MF approach and simulation results.

Figure 3 shows plots of the phase boundary for both the $A$ and $B$ saturated phases obtained by simulations, pair, and three-sites MF approximations. Within the simple MF analysis the phase boundary for the $A(B)$ saturated phase always occurs at $p=1$ $(q=1)$, that is why it is not included in the figure. For weak repulsions, the pair MF approach gives better results for both the $A$ and $B$ phase boundaries than the three-sites MF analysis. However, for stronger repulsions, correlations become more important and the three-sites MF approximation leads to better results. It should be pointed out that no bicritical point can be obtained from the MF approximations considered in this work. However, it is observed in figure 4 that phase boundary curves qualitatively resemble the actual phase diagram. The closest points in the phase diagram obtained within the three-site MF approach are $\left(r_{c} \approx 0.6, p_{c}^{A} \approx 0.348\right),\left(r_{c} \approx 0.6, p_{c}^{B} \approx 0.305\right)$ which are good approximations for the actual bicritical point $\left(r_{c}=0.559, p_{c}=0.35\right)$ (see next section). In the MF treatment of the interacting monomer-monomer reaction model 21] a bicritical point can always be found given the symmetry of the adsorption process which is reflected in the MF rate equations. However, no good approximation for this point was obtained up to the three-sites MF analysis.

The monomer-dimer reaction model with lateral interactions displays a feature that is not present in the interacting monomer-monomer reaction model. In fact, for $q=1$, the stationary density $\rho_{B}$ is always less than one in spite of the value of the repulsion $r$. However, $\rho_{B}$ is a function of $r$ since local configurations like $B B V B B$ are more likely to occur when the repulsion is increased. Then, for $q=1$, we have a one-dimensional random dimer filling problem with lateral interactions. In this case $\rho_{B}$ is commonly called jamming coverage which we denote as $\Theta_{j}(r)$.

Figure 5 shows a plot of $\Theta_{j}(r)$ versus $r$ obtained by simulations, pair and three-sites 
MF approximations. It should be noted that a MF analysis of the jamming coverage requires at least to take into account correlations up to pairs of sites. For $r=0$ both MF analysis predict $\Theta_{j}(0)=0.8646 \ldots$, reproducing the value derived long ago by Flory [24]. As it has also been observed for the phase boundary curves, the three-sites (pair) MF approximation gives better results for strong (weak) repulsions.

We did not calculate higher order MF approximations since the algebra becomes much more complicated and the MF approaches presented here provide a fairly good qualitative understanding of the model.

\section{Simulations Results}

As mentioned in the last section, static Monte Carlo simulations are suitable to obtain the coordinates of the first-order transition points. However, second-order IPT are dominated by fluctuations, so in a finite system and close to the critical point, the stationary states of the reactive phase can irreversibly evolve into the saturated state (absorbing state). Due to this circumstance, the precise determination of both critical points and critical exponents is rather difficult. However, this shortcoming can be avoided by performing an epidemic analysis [1]. Within this context, the epidemic analysis is usually called "defect dynamics" simulations. For this purpose one starts, at $t=0$, with a small block of vacant sites in an otherwise saturated lattice, i.e. a configuration close to one of the absorbing states. Then, the time evolution of this block is analyzed by measuring the following properties: (i) The average number of vacant sites at time $t, N(t)$, (ii) the survival probability of the block at time $t, P(t)$, and (iii) the average distance over which the block has spread at time $t, R(t)$. Finitesize effects are absent because the system is taken large enough to avoid the presence of vacant sites at the boundaries. For this purpose a lattice of $10^{4}$ cells is enough. Averages are taken over $10^{5}$ different samples. Near the critical point, the number of vacant sites is often very small. Then, we improve the efficiency of the algorithm by keeping a list of vacant sites. Time is incremented by $1 / n(t)$, where $n(t)$ is the number of vacant sites at time $t$. Time evolution of blocks are monitored up to $t=10^{5}$. At criticality, the following scaling behavior holds:

$$
\begin{gathered}
N(t) \propto t^{\eta}, \\
P(t) \propto t^{-\delta},
\end{gathered}
$$


and

$$
R(t) \propto t^{z / 2}
$$

where $\delta, \eta$ and $z$ are dynamic exponents.

At the bicritical point, it is useful to perform another epidemic analysis often called "interface dynamics" simulations. In this case one starts at $t=0$ with a minimum width interface between two saturated phases. Since $A B$ pairs immediately react, there must always be at least a vacancy between two saturated phases. Then, the epidemic always survives and consequently $\delta=0$. In addition, a second type of "interface dynamics" simulation can also be performed. In this case the simulation finishes when the interface has collapsed back to its initial width [19, 21]. At the bicritical point equations (16), (17), and (18) hold but $P(t)$ must be interpreted as the probability that the interface has not returned to its minimum width. Interface dynamics simulations give us information about the competitive growth of different domains.

Figure 6 shows log-log plots of $N(t), P(t)$, and $R(t)$ for different values of $p$ close to the phase boundary between the $B$-saturated and the reactive phase keeping $r=0.9$ constant. The straight line obtained for the three quantities mentioned above at $q=$ 0.835 is the signature of critical behavior, while slight upward (downward) deviations for $q=0.8325$ ( $q=0.8375)$ indicate supercritical (sub-critical) behavior, respectively. In this way we have determined the critical points along both second order phase boundary curves. The analysis at the bicritical point is discussed later. The spreading or epidemic analysis is a powerful method since the error bars for the calculations of the critical points are on the third digit. The critical exponents obtained at various critical points along the phase boundary curves have the same values (within error bars) and are in good agreement with the dynamic critical exponents corresponding to DP which are the following:

$$
\delta \cong 0.162, \eta \cong 0.317, z \cong 1.282
$$

By drawing the second order phase boundary curves one gets the first clue to the position of the bicritical point. The main property of the system at the bicritical point is the symmetry of both saturated phases. This means that at the bicritical point both absorbing states are statistically equivalent. Then, defect dynamics simulations at the bicritical point should give identical results no matter what saturated phase is used to 
start the simulation.

Figure 7 shows log-log plots of $N(t), P(t)$, and $R(t)$ obtained from defect dynamics simulations started using different absorbing states at the bicritical point. It is observed that the critical behavior up to time $t \approx 10^{5}$ is governed by the same exponents. Then, our best estimation of the value of the bicritical point is $\left(r_{c}=0.559, p_{c}=0.35\right)$. It should be pointed out that in the study of the interacting monomer-monomer reaction model 21] the localization of the bicritical point was easier because of the symmetry of the adsorption-reaction processes.

We obtain the following values for the dynamical critical exponents at the bicritical point

$$
\delta=0.2910 \pm 0.0002, \eta=0.0034 \pm 0.0003, z=1.147 \pm 0.0004
$$

It should be remarked that the error bars merely indicate the statistical errors obtained from regressions.

The exponent $\beta$ which characterizes the critical behavior of the order parameter has been obtained directly in static simulations. For the present model a good choice for the order parameter is the average density of empty sites $\rho_{V}$. The behavior of the order parameter close to a critical point is given by

$$
\rho_{V} \sim|\Delta|^{\beta}
$$

where $\Delta$ is the distance from a point within the reactive phase to the bicritical point. Figure 8 shows a plot of $\rho_{V}$ versus $\Delta$ where the points within the reactive phase belong to a straight line that bisects the reactive window. Although static simulations are known to be quite inaccurate to determine $\beta$, we found the reasonable value $\beta=0.88 \pm 0.005$.

The set of critical exponents calculated at the bicritical point are in good agreement with the ones corresponding to the PC universality class. For defect dynamics simulations at $r>r_{c}$ and close to the bicritical point a crossover is observed where the critical behavior at short times (long times) is governed by the PC (DP) universality class.

In the following we present the results of the "interface dynamics" simulations. Figure 9 shows plots of the number of vacancies in the interface $N(t)$ and the average size of the interface $R(t)$ obtained using the first type of "interface dynamics" simulation 
(where $\delta=0$ ). We average over $10^{5}$ independent samples and we find the following value for the dynamical critical exponents:

$$
\eta_{1}=0.2840 \pm 0.0002, z_{1}=1.1506 \pm 0.0004
$$

This values are in good agreement with the corresponding critical exponents obtained in other models in the PC universality class [13, 14, 15, 18, 19]. It should be noted that the values of $\eta_{1}$ and $z_{1}$ are similar to the ones obtained from "defect dynamics" simulations if we only average over surviving runs. Then the first type of "interface dynamics" simulation yields no new information about the dynamics of the interface and reflects the full equivalence of the absorbing states at the bicritical point.

Figure 10 shows plots of $N(t), P(t)$, and $R(t)$ obtained using the second kind of "interface dynamics" simulation. We average over $9 \times 10^{6}$ independent samples and we find the following values for the critical exponents:

$$
\delta_{2}=0.7163 \pm 0.0003, \eta_{2}=-0.4277 \pm 0.0004, z_{2}=1.160 \pm 0.0006
$$

We first observe that the values of the exponents $z_{1}$ and $z_{2}$ are similar. The exponent $\eta_{1}$ must be compared with $\delta_{2}+\eta_{2}$ which governs the time evolution of the vacant sites averaged only over the surviving runs. Both exponents are equal within error bars. This is the signature of the universal behavior of the critical spreading of the interface. The value of the new independent dynamical exponent $\delta_{2}$ supports the Bassler-Browne conjecture [19] which states that $\delta_{2}$ is a new universal exponent within the PC class. Recently Park and Park 22] have found that the interacting monomer-dimer reaction model with infinite repulsive interaction $(r=1)$ supports a kink representation where the total number of kinks is conserved modulo 2. By including a parity-conserving symmetry breaking field that favors one of the absorbing states, the authors showed that the critical behavior of the model changes from PC to DP. Then, they concluded that the conservation of the number of kinks modulo 2 is not the reason for observing a universality class different from DP but the symmetry of the absorbing states.

In contrast to the case with infinite repulsion, the present variant of the model involve adsorption-reaction processes in which parity is not conserved explicitly. Although by their own nature the number of domain walls are conserved modulo 2 , it should be remarked that a non-trivial PC dynamics requires the creation of at least three kinks per step which is not possible in our model. This corroborates that the 
symmetry among absorbing states is indeed the only essential property of models in the PC class [13, 18, 19, 21].

It is interesting to discuss the relation between the present model and the branching and annihilating walks models. Static Monte Carlo simulations close to the bicritical point reveal that stationary configurations are formed by large clusters of different species that survive a long time. Then, it is clear that the dynamics of domain walls between clusters of different species will be relevant at the bicritical point. Following the ideas presented in reference [18], at the bicritical point "walkers" can be identified with domain walls between clusters of different species. It should be pointed out that we have to generalize the concept of walkers. In fact, the "walkers" defined above are extended objects of fluctuating width. It should be noticed that at the bicritical point the typical width of a walker is only few lattice sites. Considering the time evolution of this "walkers" in the long-time regime and for large lattices, an effective parityconserving dynamics may be restored. Concerning the dynamics of these "walkers", it is possible to identify an inactive and an active phase. In fact, the line defined by the first-order phase transition points, corresponds to the inactive phase for the dynamics of the "walkers", which ends at the bicritical point. A continuation of this line from the bicritical point through the reactive phase, would correspond to the active phase for the dynamics (see figure 1). In other words, the dynamics of this walkers is defined within a subspace of the phase diagram in which the statistical weight of the kinetic processes involving the species $A$ and $B$ is the same. Recently, Cardy and Täuber have developed a systematic theory for the branching and annihilating random walkers [25]. The authors have shown that in one dimension, fluctuation effects lead to the emergence of a non-trivial inactive phase for values of the branching rate $0 \leq \sigma<\sigma_{c}$, and a dynamic phase transition at $\sigma_{c}>0$, in contrast to the mean-field result $\sigma_{c}=0$. However, in two dimensions the theory predicts that fluctuation effects generate logarithmic corrections and the critical branching rate takes the value predicted by the bare meanfield theory $\left(\sigma_{c}=0\right)$. Then, the identification of an inactive phase for $0 \leq r<r_{c}$ and a dynamic phase transition at $r_{c}>0$ in the present model, is in complete agreement with the theoretical results. Recent results for a two-dimensional monomer-monomer surface-reaction model with repulsive lateral interactions 26] indicate that the critical branching rate takes the value $r_{c}=0$ (mean-field value), also supporting the theoretical results. 
The same ideas can also be applied to other models in the PC universality class that violate local parity conservation [19, 21].

\section{Conclusions}

We have studied a monomer-dimer surface reaction lattice model with lateral repulsion among same species using a mean-field analysis and Monte Carlo simulations. For weak repulsions the model exhibits first-order IPT between two phases saturated by different species. Increasing the interaction a reactive window appears whose edges are secondorder critical lines that separate this state from the absorbing states.

We have considered MF approximations that take into account correlations up to three lattice sites. The stationary density of the species, the phase boundary curves, and the jamming coverage have been studied within the MF approaches and the results compared with simulations. For weak (strong) repulsion the pair (three-sites) MF approximation has led us to better results than the three-sites (pair) MF approximation for all the quantities mentioned above. No bicritical point has been obtained from the MF approximations considered in this work. However, phase boundary curves calculated within MF approximations qualitatively resemble the actual phase diagram. Within the three-sites MF approach phase boundary curves come up closest at $\left(r_{c} \approx\right.$ $\left.0.6, p_{c}^{A} \approx 0.348\right),\left(r_{c} \approx 0.6, p_{c}^{B} \approx 0.305\right)$ which are good estimations for the actual bicritical point $\left(r_{c}=0.559, p_{c}=0.35\right)$.

The critical behavior of the model has been studied using both static and dynamical Monte Carlo simulations. IPT between the reactive phase and any of the saturated phases are in the DP universality class. However, it has been found a critical behavior different from DP at the point where both DP curves meet. The critical exponents calculated at the bicritical point are in good agreement with the ones corresponding to the PC class. Since this model does not conserve explicitly any quantity modulo 2 , it corroborates that the twofold symmetry in the absorbing states is the reason for obtaining a critical behavior different from DP. It is relevant to mention that the authors of the theory for the branching and annihilating random walkers [25] have identified a formal permutation symmetry at the Hamiltonian level in the case of an even number of offsprings. This fact again indicates that symmetry among absorbing states plays a relevant role in determining the universality class.

The value of the critical exponent $\delta_{2} \cong 0.71$ corresponding to the probability $P(t)$ 
that the interface has not collapsed back to its minimum width is in good agreement with the values obtained in other models [19, 21, 27]. This corroborates the BasslerBrown conjecture [19] which states that the interfacial fluctuations are an additional universal characteristic of models in the PC class.

Acknowledgments: I would like to thank Uwe Täuber and Ezequiel Albano for a careful reading of the manuscript. Interesting discussions with Uwe Täuber and Haye Hinrichsen are also acknowledged. This work was financially supported by CONICET (Argentina).

\section{References}

[1] P. Grassberger and A. de la Torre, Ann. Phys. (New York) 122, 373 (1979); P. Grassberger, J. Phys A: Math. Gen. 22, 3673 (1989).

[2] M. Moshe, Phys. Rep. C 37, 255 (1978).

[3] R. Ziff, E. Gulari, and Y. Barshad, Phys. Rev. Lett. 56, 2553 (1986).

[4] I. Jensen, H. Fogedby, and R. Dickman, Phys. Rev. A 41, R3411 (1990).

[5] E. V. Albano, Phys. Rev. Lett. 69, 656 (1992).

[6] I. Jensen, Phys. Rev. Lett. 70, 1465 (1993).

[7] H. Takayashu and A. Y. Tretyakov, Phys. Rev. Lett. 68, 3060 (1992); I. Jensen, Phys. Rev. E 47, 1 (1993).

[8] H. K. Janssen, Z. Phys. B 42, 151 (1981); P. Grassberger, Z. Phys. B 47, 365 (1982).

[9] G. Grinstein, Z. W. Lai and D. Browne, Phys. Rev. A 40, 4820 (1989); I. Jensen and R. Dickman, Phys. Rev. E 48, 1710 (1993); I. Jensen, J. Phys. A: Math. Gen. 27, L61 (1994).

[10] P. Grassberger, F. Krause and T. von der Twer, J. Phys. A Math. Gen. 17, L105 (1984).

[11] P. Grassberger, J. Phys. A Math. Gen. 22, L1103 (1984). 
[12] N. Menyhárd, J. Phys. A Math. Gen. 27, 6139 (1994); N. Menyhárd and G. Ódor, J. Phys. A Math. Gen. 29, 7739 (1996).

[13] M. H. Kim and H. Park, Phys. Rev. Lett. 73, 2579 (1994); H. Park, M. H. Kim, and H. Park, Phys. Rev. E 52, 5664 (1995); M. H. Kim and H. Park, J. Korean Phys. Soc. 26, S345 (1993).

[14] D. ben-Avraham, F. Leyvraz, and S. Redner, Phys Rev. E 50, 1843 (1994).

[15] I. Jensen, J. Phys. A Math. Gen. 26, 3921 (1993).

[16] I. Jensen, Phys. Rev. E 50, 3623 (1994).

[17] U. Täuber, M. Howard, and H. Hinrichsen, cond-matt 9709057, (1997); U. Alon, M. R. Evans, H. Hinrichsen, and D. Mukamel, Phys. Rev. Lett. 76, 2746 (1996).

[18] H. Hinrichsen, Phys. Rev E 55, 219 (1997).

[19] K. E. Bassler and D. A. Brown, Phys. Rev. Lett. 77, 4094 (1996); Phys. Rev. E $55,5225(1997)$.

[20] J. Zhuo, S. Redner, and H. Park, J. Phys. A: Math. Gen. 26, 4197 (1993).

[21] K. S. Brown, K. E. Bassler and D. A. Brown, Phys. Rev E 56, 3953 (1997).

[22] H. Park and H. Park, Physica A 221, 97 (1995).

[23] R. Dickman, Phys. Rev. A 34, 4246 (1986), H. Gutowitz, J. D. Victor, and B. W. Knight, Physica 28D, 18 (1987).

[24] P. J. Flory, J. Am. Chem. Soc. 61, 1518 (1939).

[25] J. L. Cardy and U. C. Täuber, Phys. Rev. Lett. 77, 4780 (1996); J. L. Cardy and U. C. Täuber, J. Stat. Phys. 90, 1 (1998).

[26] R. A. Monetti, in preparation.

[27] W. M. Hwang, S. Kwon, H. Park, and H. Park, cond-mat/9712259, (1997). 
Figure 1: Phase diagram of the model obtained by means of Monte Carlo simulations.

Figure 2: Plots of $\rho_{A}$ and $\rho_{B}$ versus $p$ and $q$ respectively, obtained using static Monte Carlo simulations, simple, pair, and three-sites MF approximations. a) $\rho_{A}$ versus $p$ for $r=0.5$, b) $\rho_{B}$ versus $q$ for $r=0.5$, c) $\rho_{A}$ versus $p$ for $r=0.9$, and d) $\rho_{B}$ versus $q$ for $r=0.9$.

Figure 3: Phase boundary curves obtained by means of Monte Carlo simulations, pair, and three-sites MF approximations. a) Boundary of the $A$-saturated phase, b) Boundary of the $B$-saturated phase

Figure 4: Phase boundary curves obtained within the pair, and three-sites MF approximations. a) Pair MF approximation b) Three-sites MF approximation.

Figure 5: Plot of the jamming coverage $\Theta_{j}(r)$ versus $r$ obtained using Monte Carlo simulations, pair, and three-sites MF approximations.

Figure 6: Log-log plots obtained using defect dynamics simulations keeping $r=0.9$ constant. a) $N(t)$ versus $t$ b) $P(t)$ versus $t$ c) $R(t)$ versus $t$.

Figure 7: Log-log plots obtained using defect dynamics simulations initiated using different absorbing states at the bicritical point. a) $N(t)$ versus $t$ b) $P(t)$ versus $t$ c) $R(t)$ versus $t$.

Figure 8: Plot of the density of vacant sites $\rho_{V}$ versus the distance $|\Delta|$ to the bicritical point. A straight line of slope $\beta=0.90$ has been included.

Figure 9: Log-log plots obtained using the first type of interface dynamics simulations $(\delta=0)$ for the following values of the parameters: $(r=0.57, p=0.3484)$ (upper curve), $(r=0.559, p=0.35)$ (middle curve) $(r=0.559, p=0.355)$ (lower curve). a) $N(t)$ versus $t \mathrm{~b}) R(t)$ versus $t$.

Figure 10: Log-log plots obtained using the second type of interface dynamics simulations for the following values of the parameters: $(r=0.57, p=0.3484)$ (upper curve), $(r=0.559, p=0.35)$ (middle curve) $(r=0.559, p=0.355)$ (lower curve). a) $N(t)$ versus $t$ b) $P(t)$ versus $t$ c) $R(t)$ versus $t$. The inset amplifies the last decade in order to distinguish between the three curves since $R(t)$ is less sensitive to changes in the parameters. 


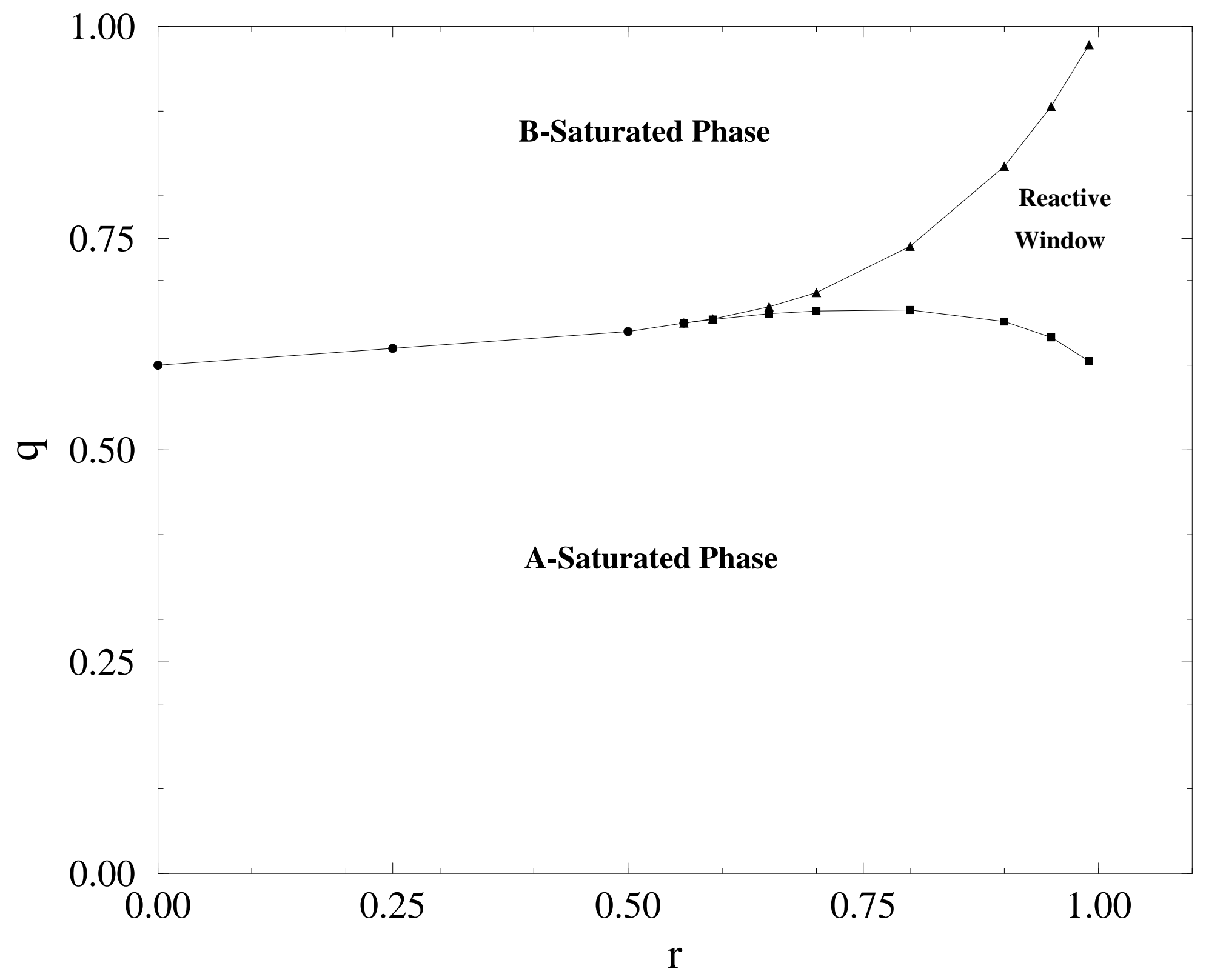




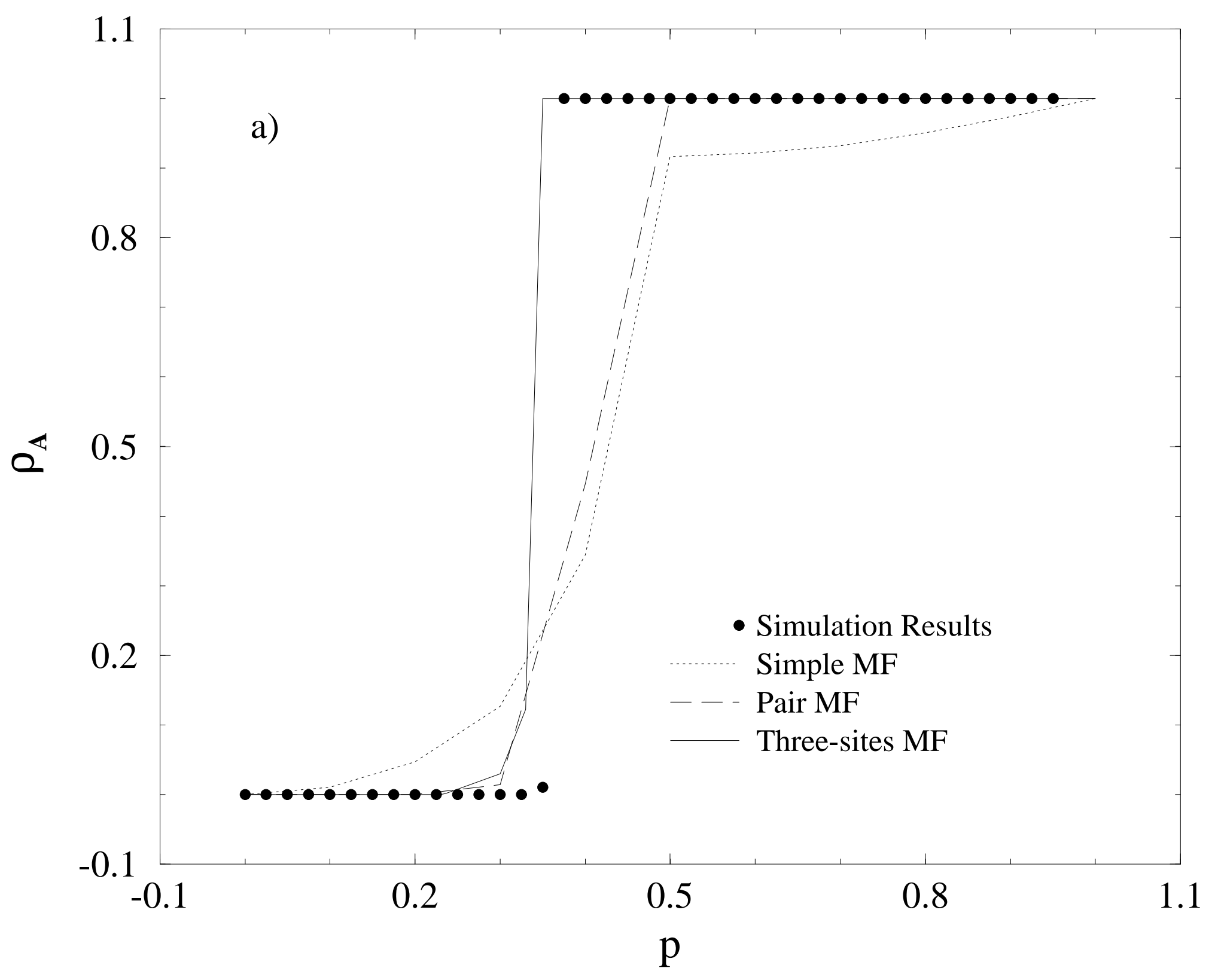




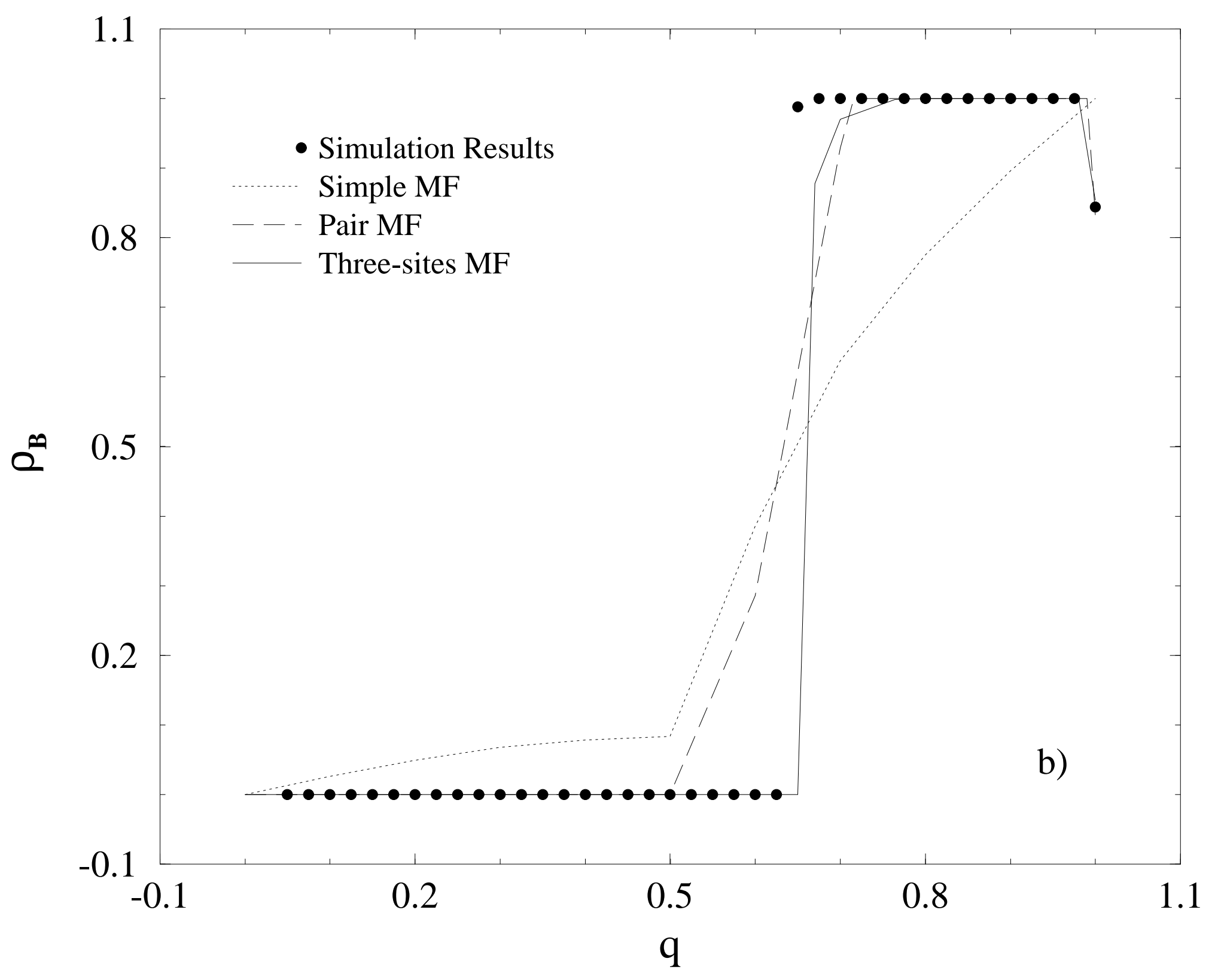




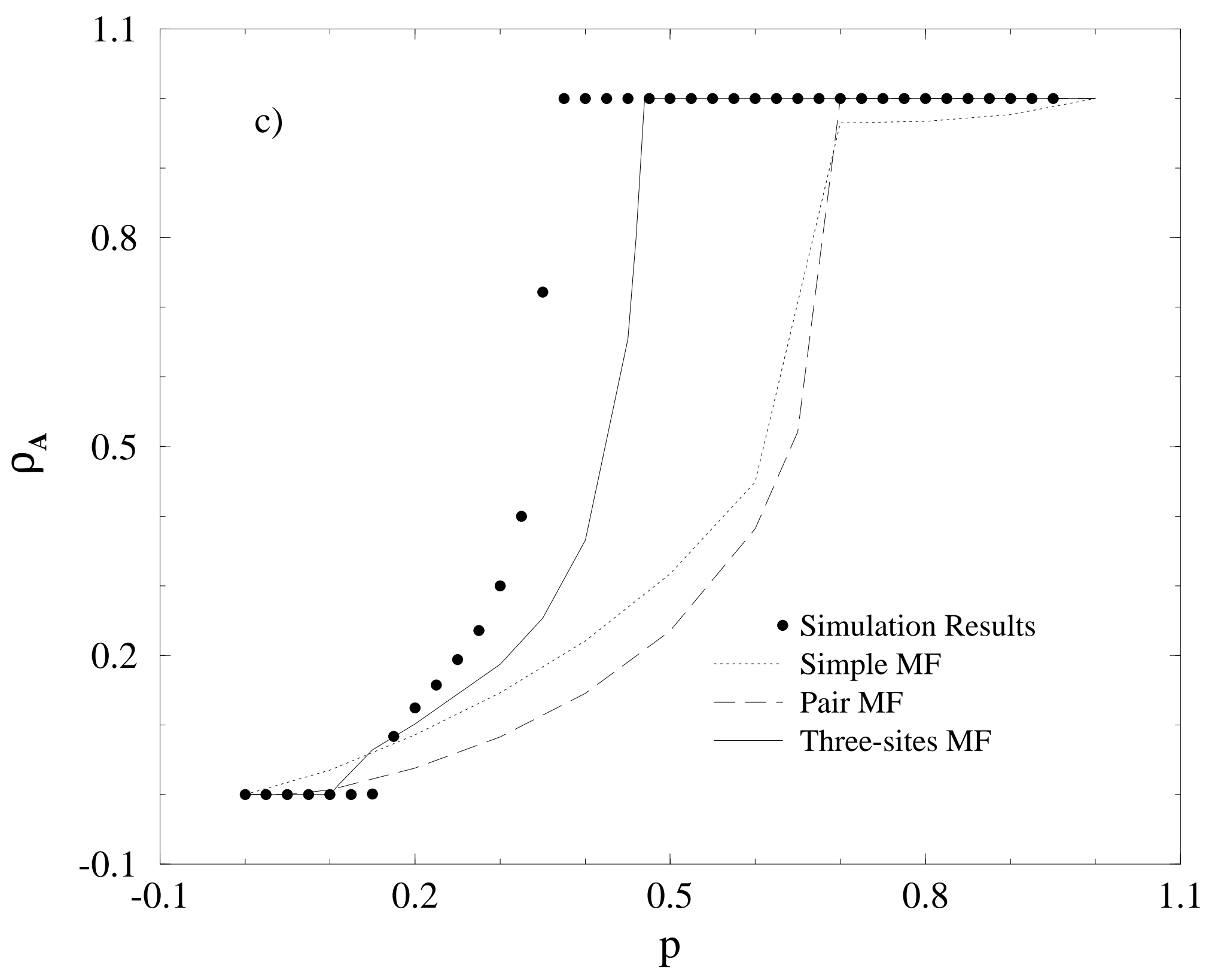




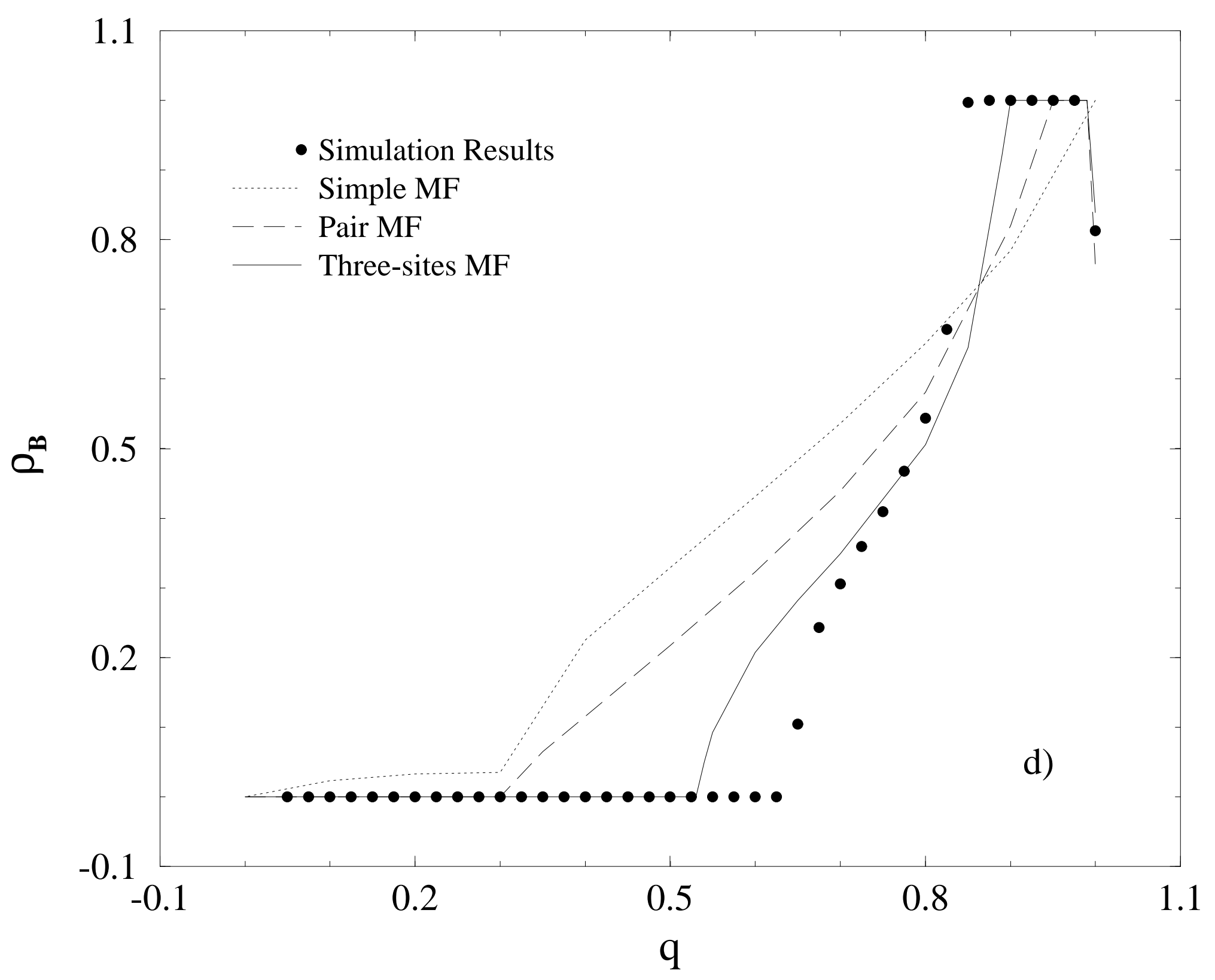




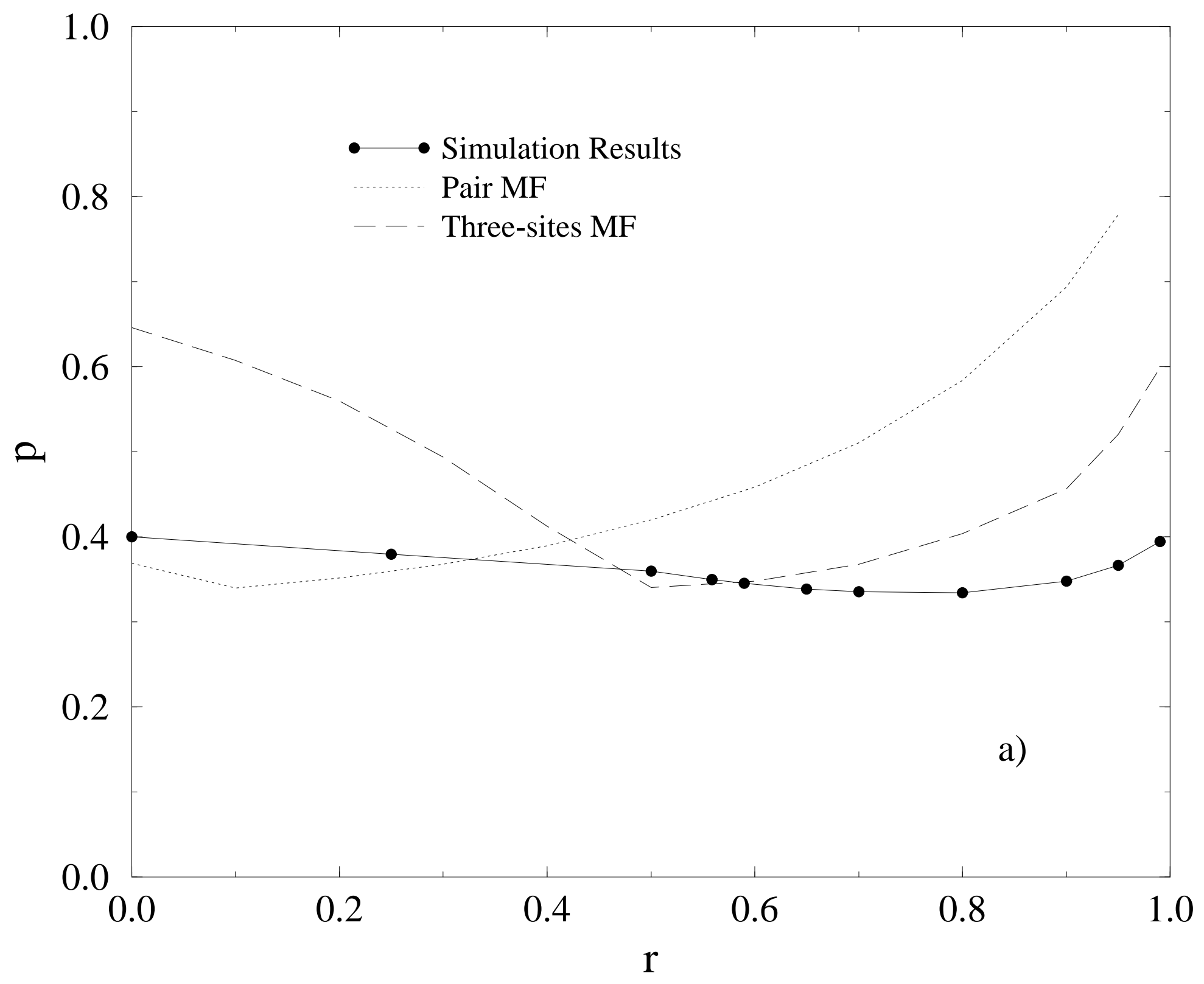




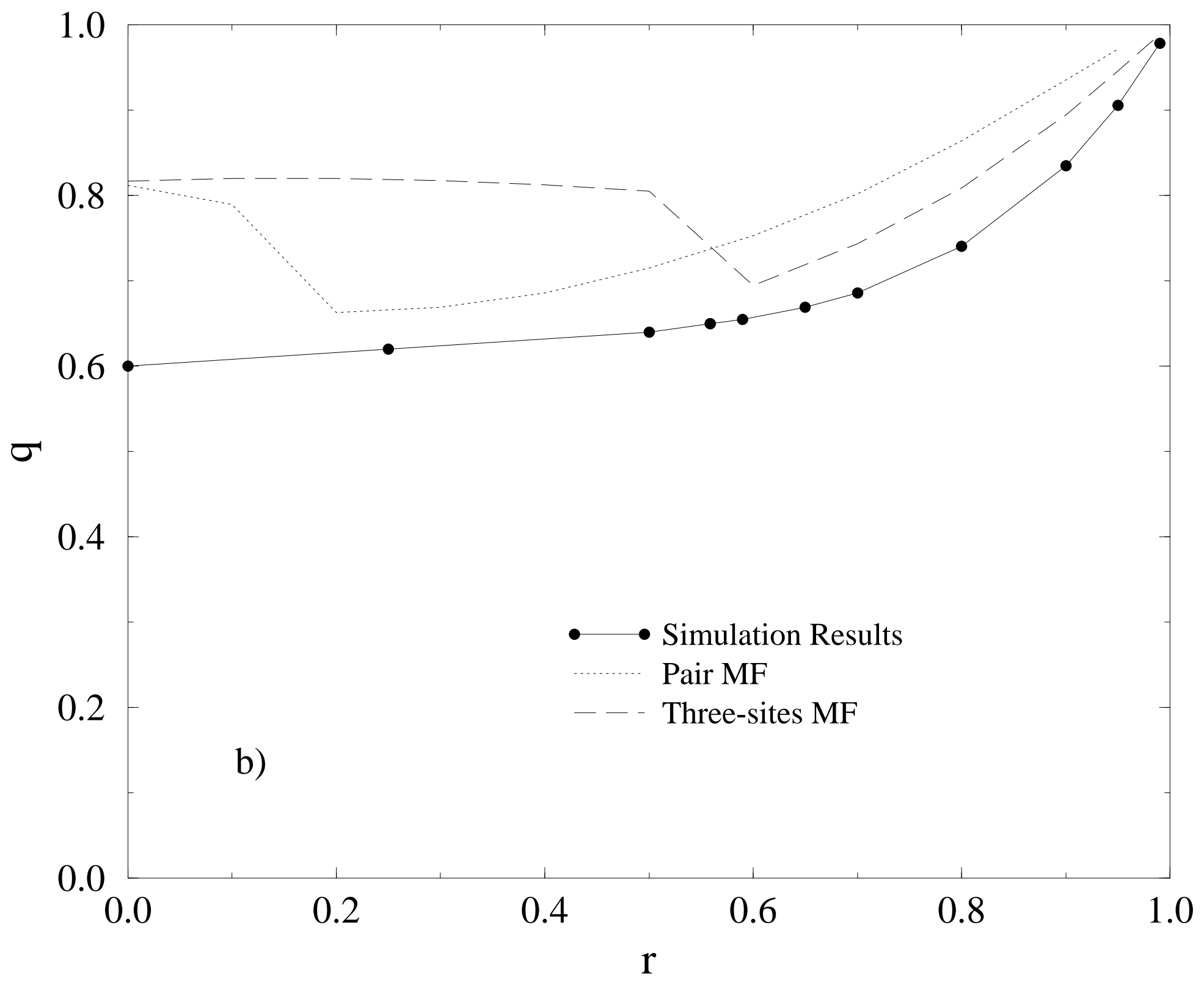




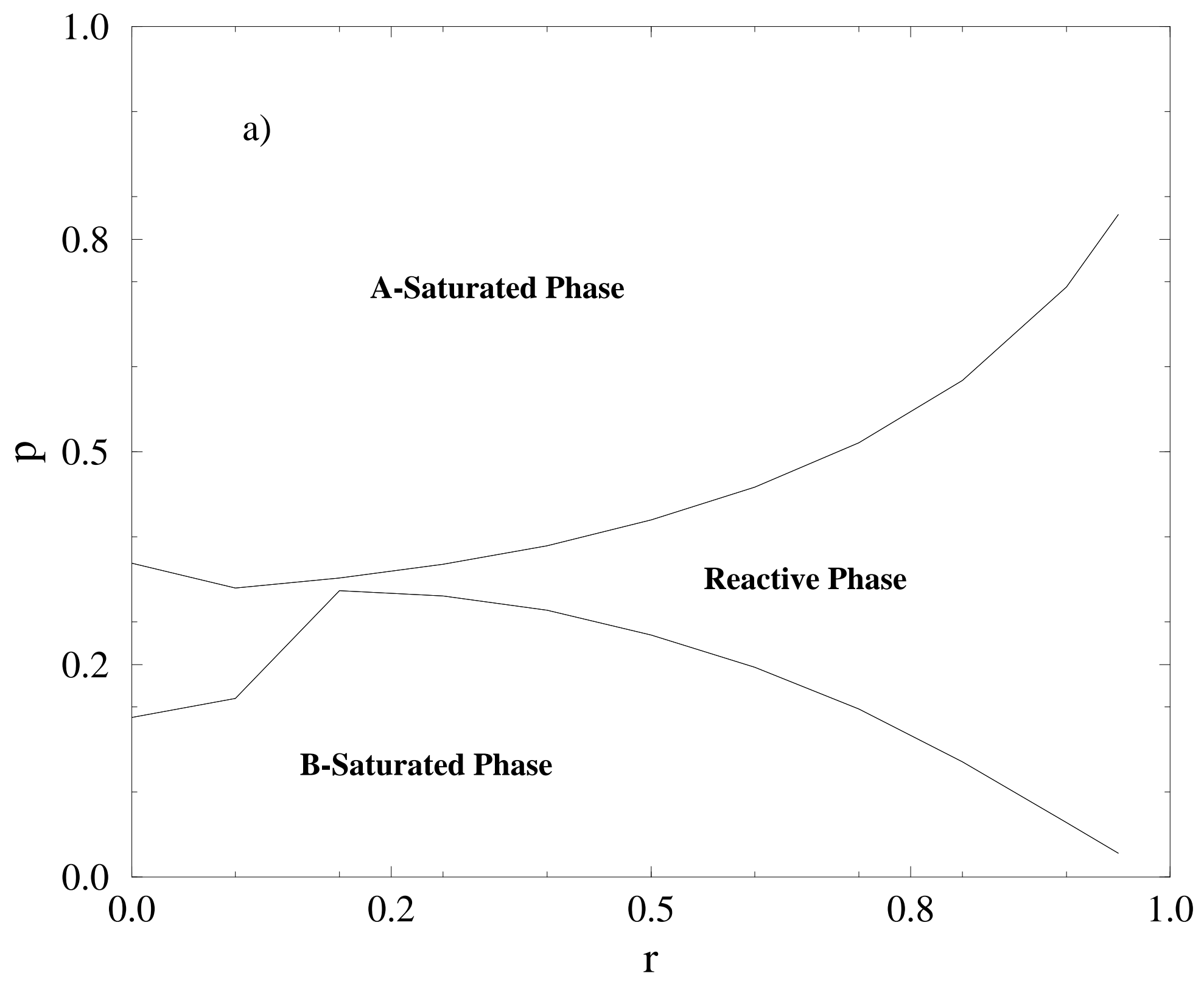




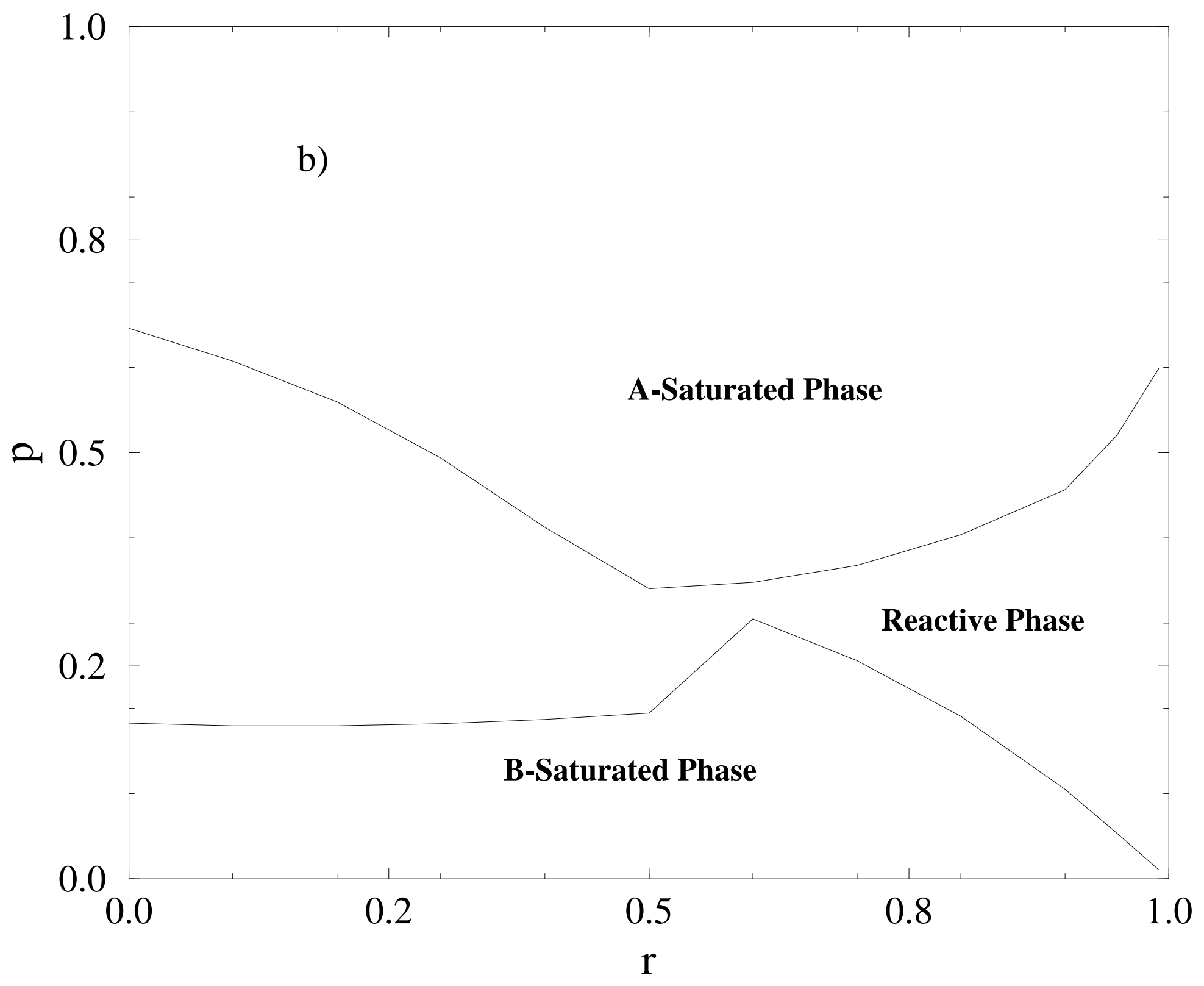




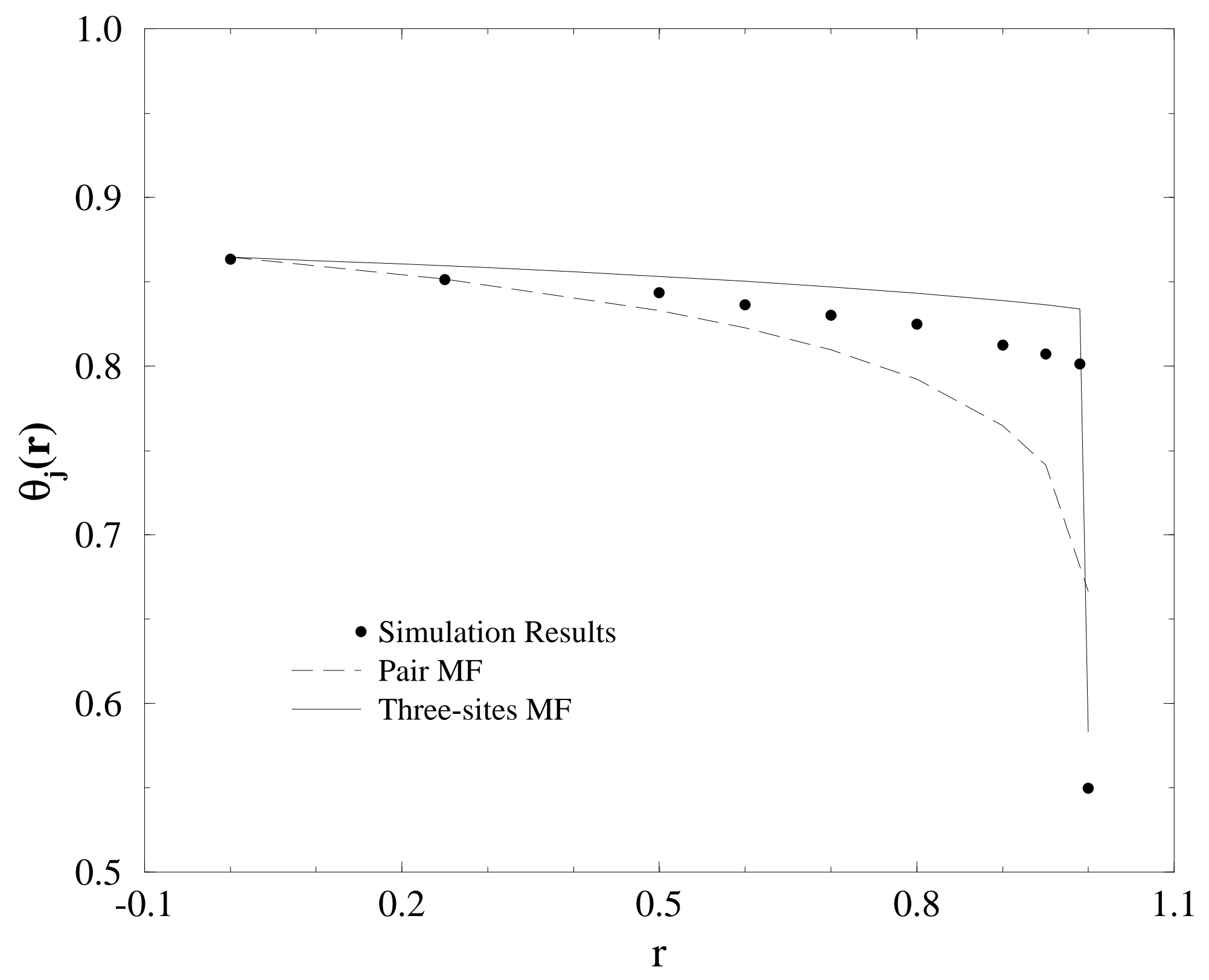




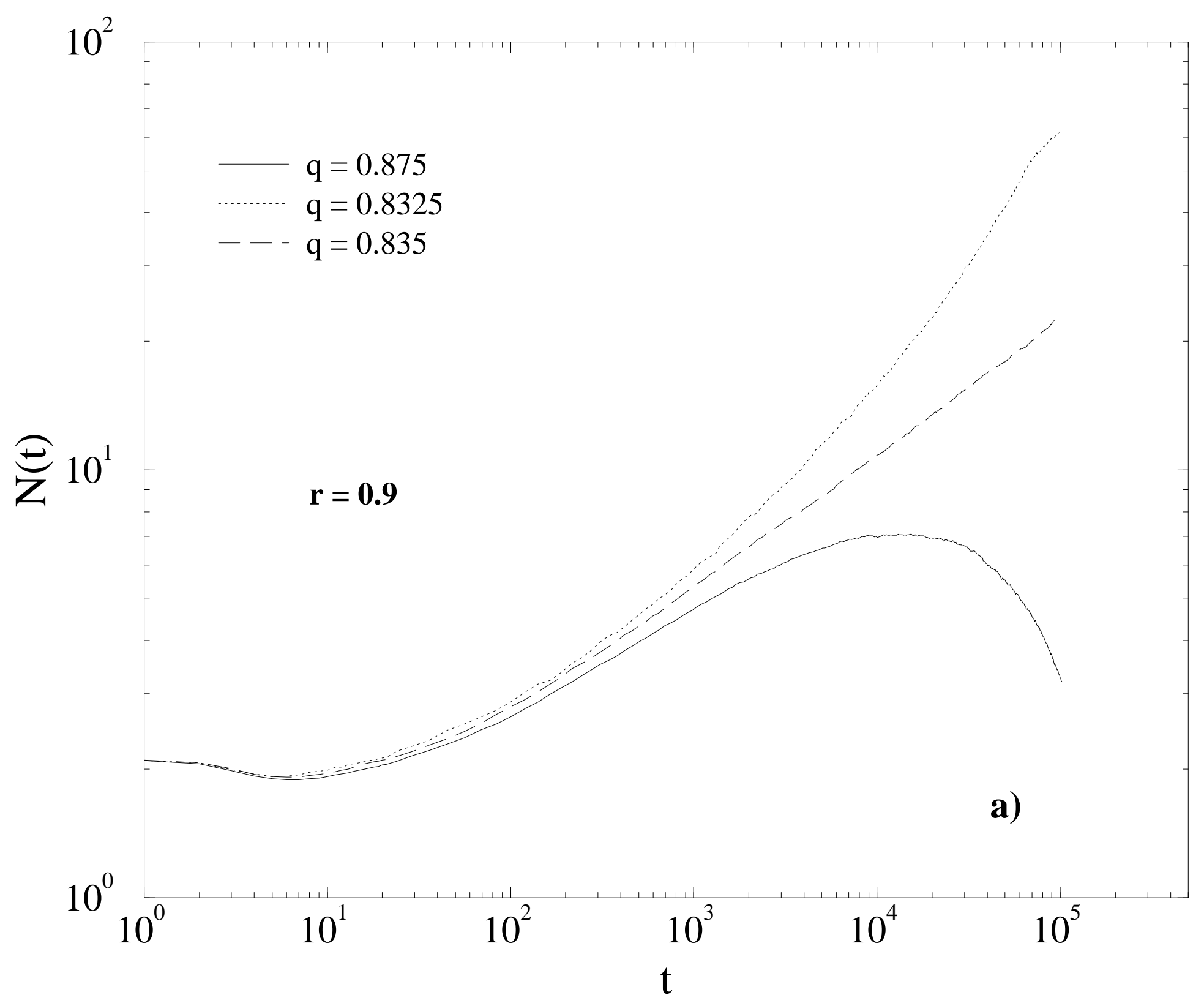




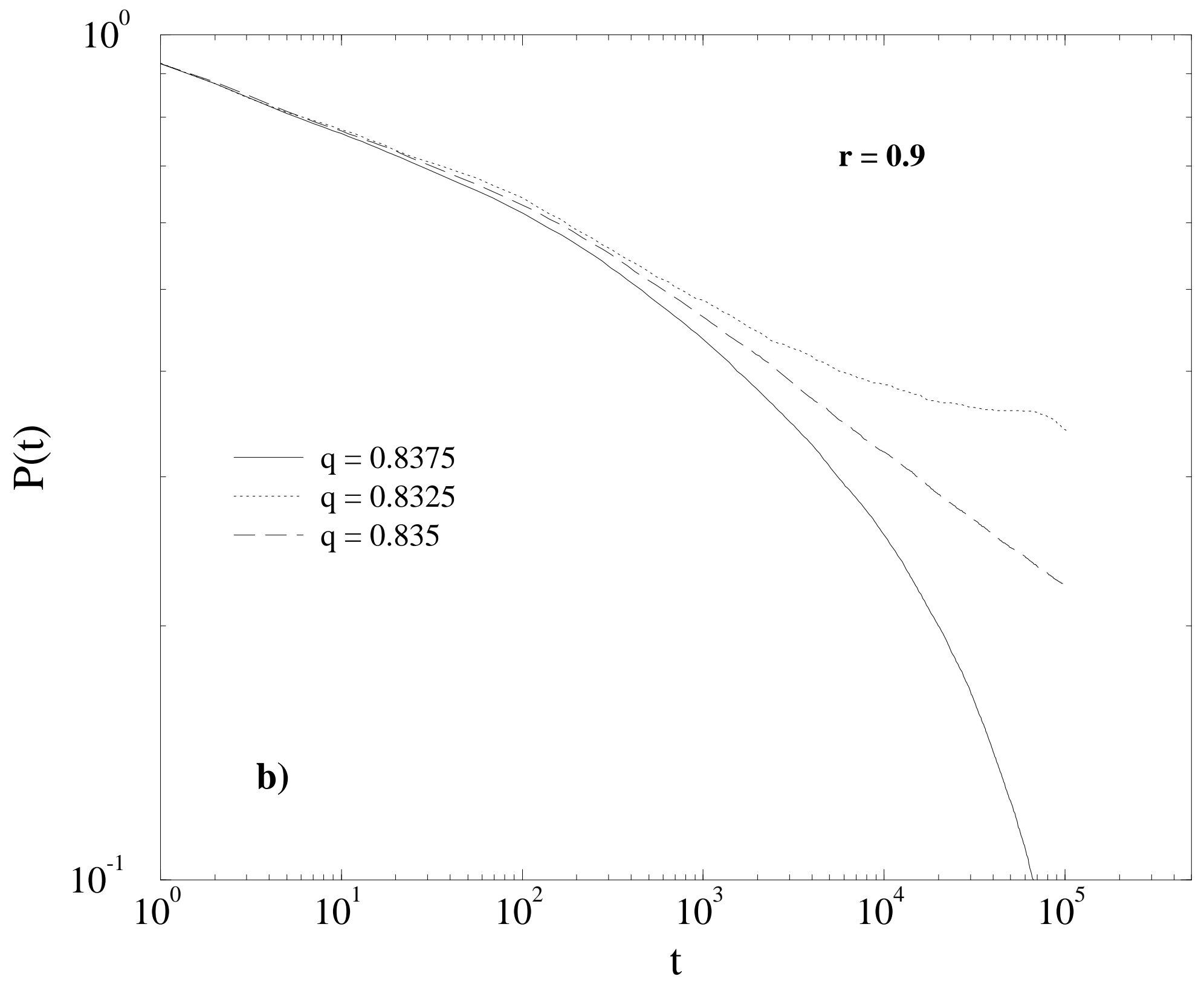




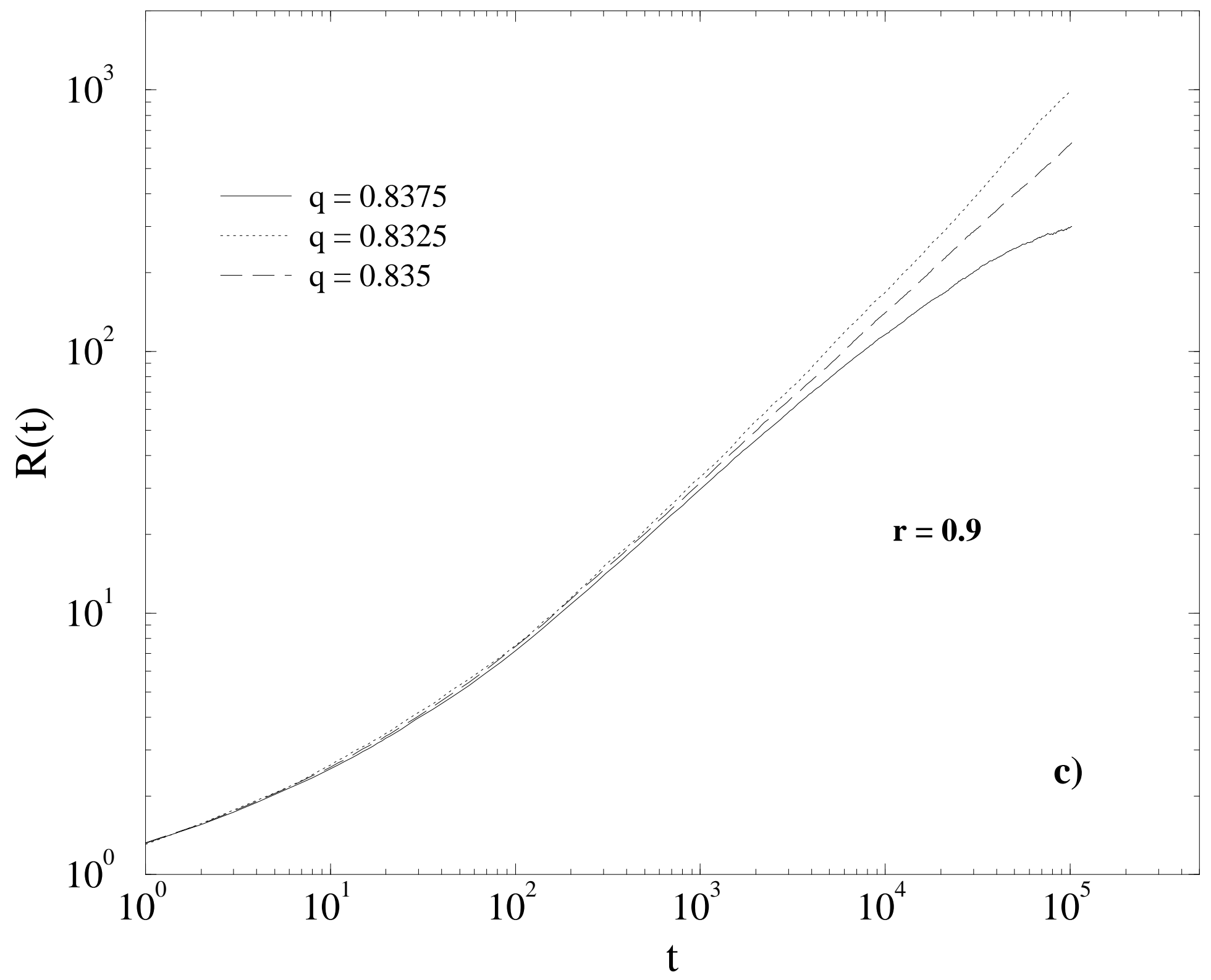




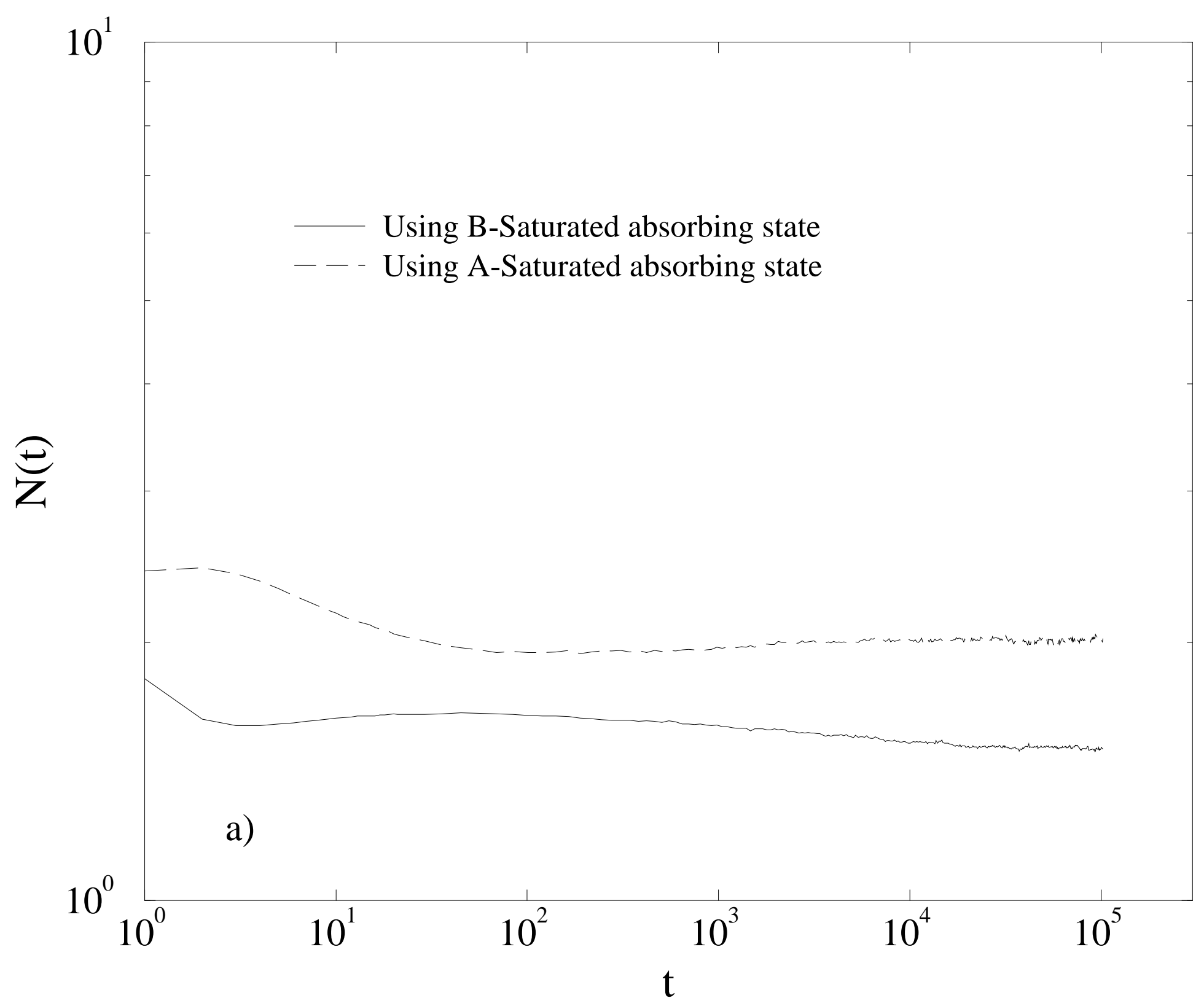




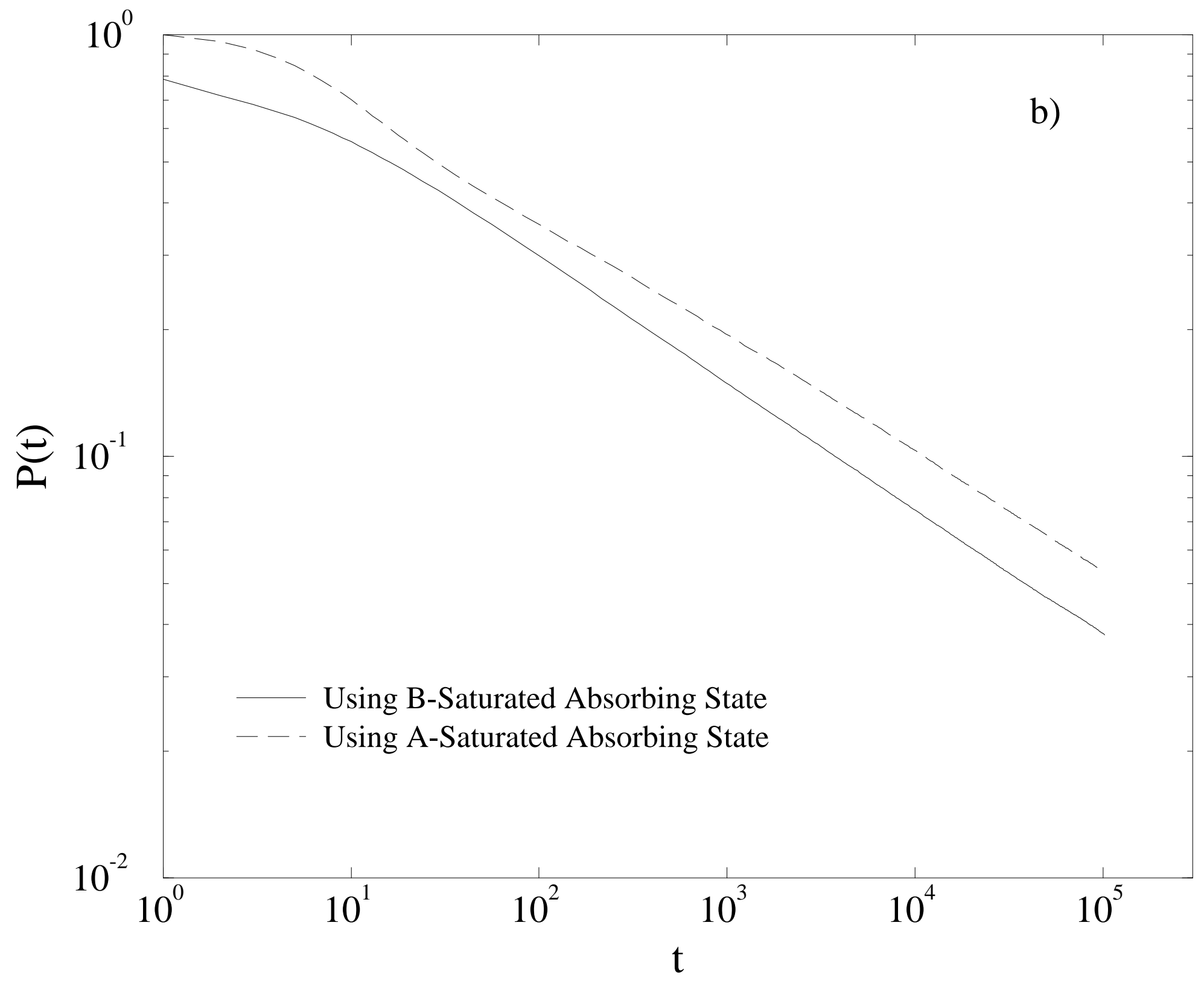




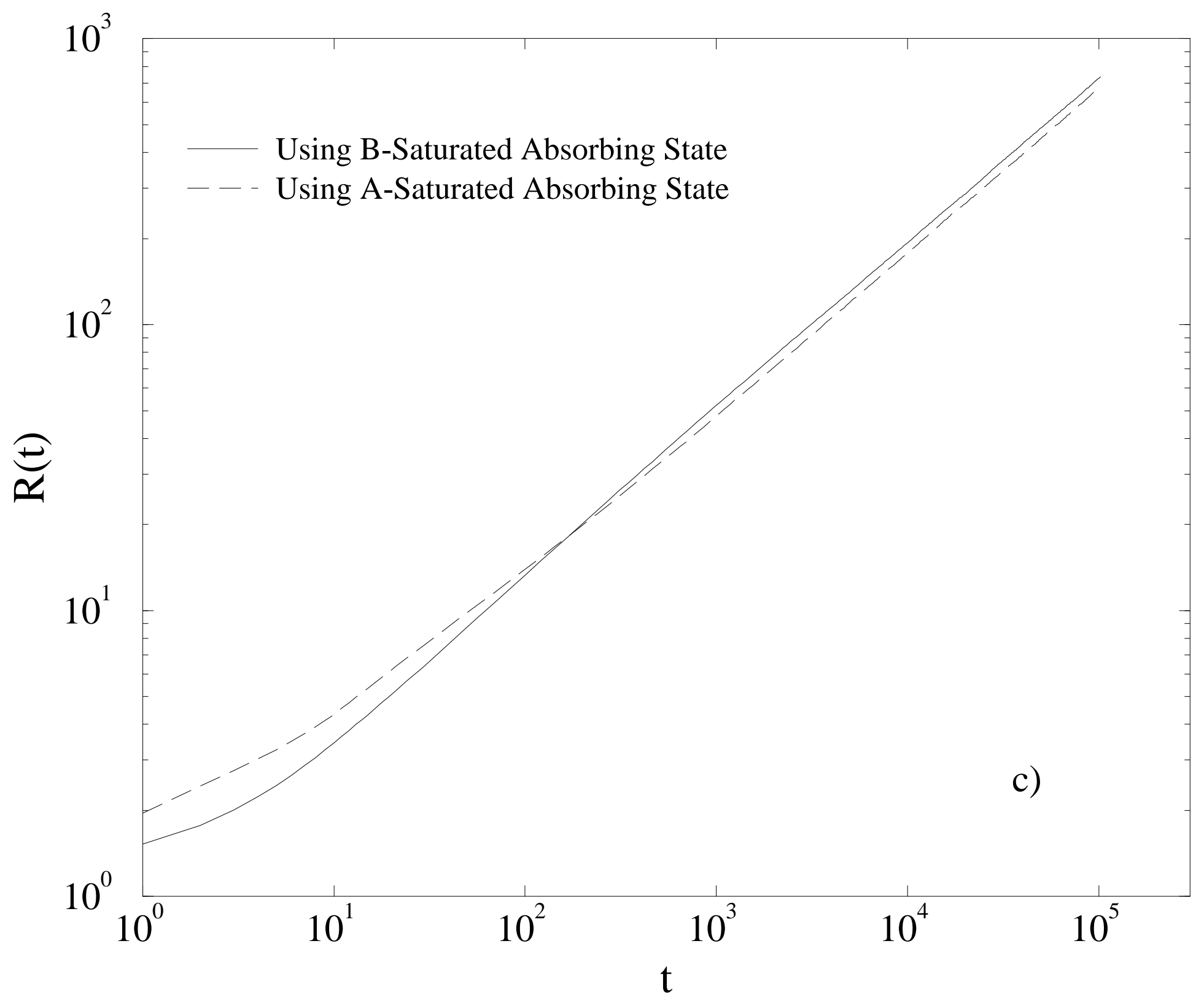




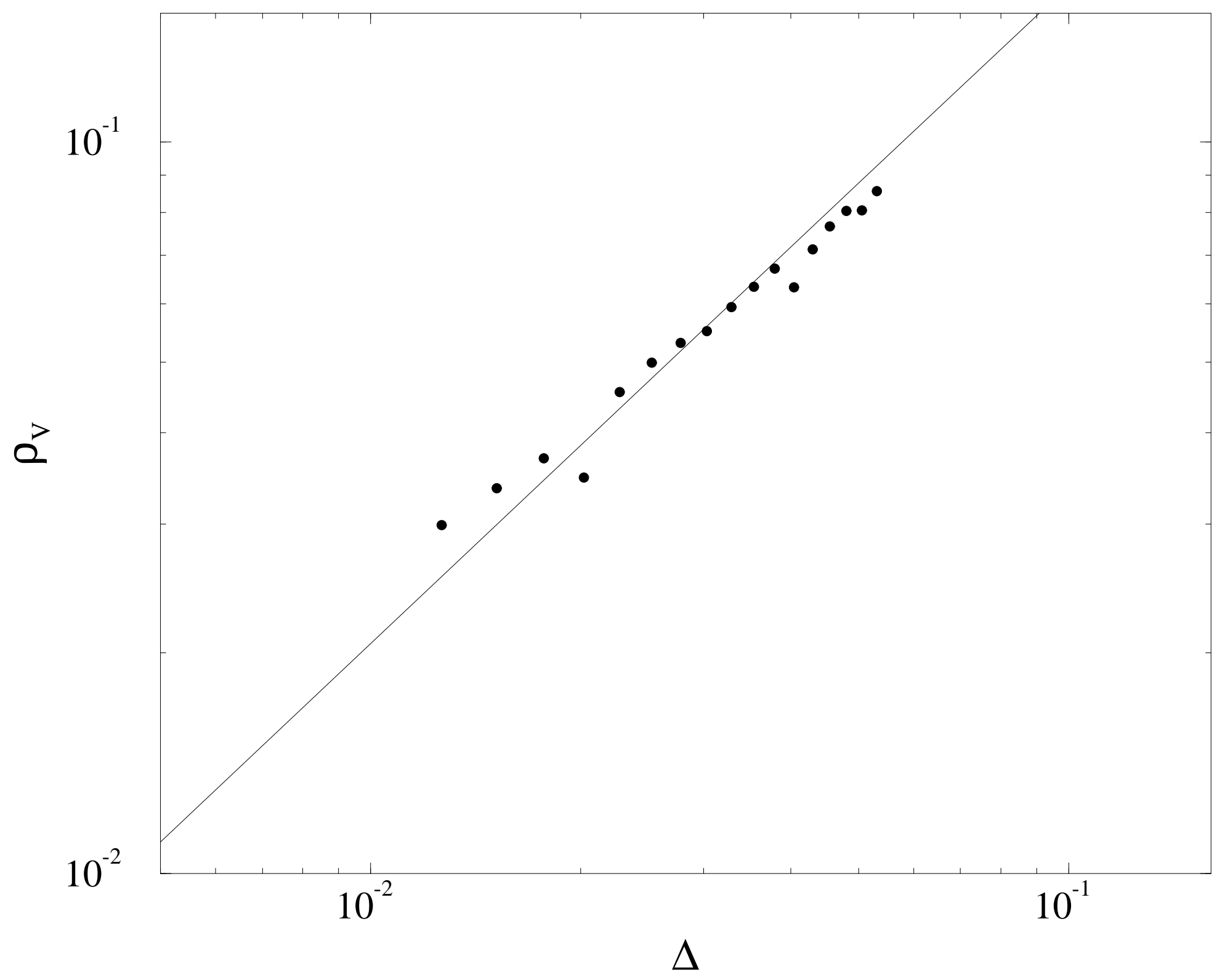




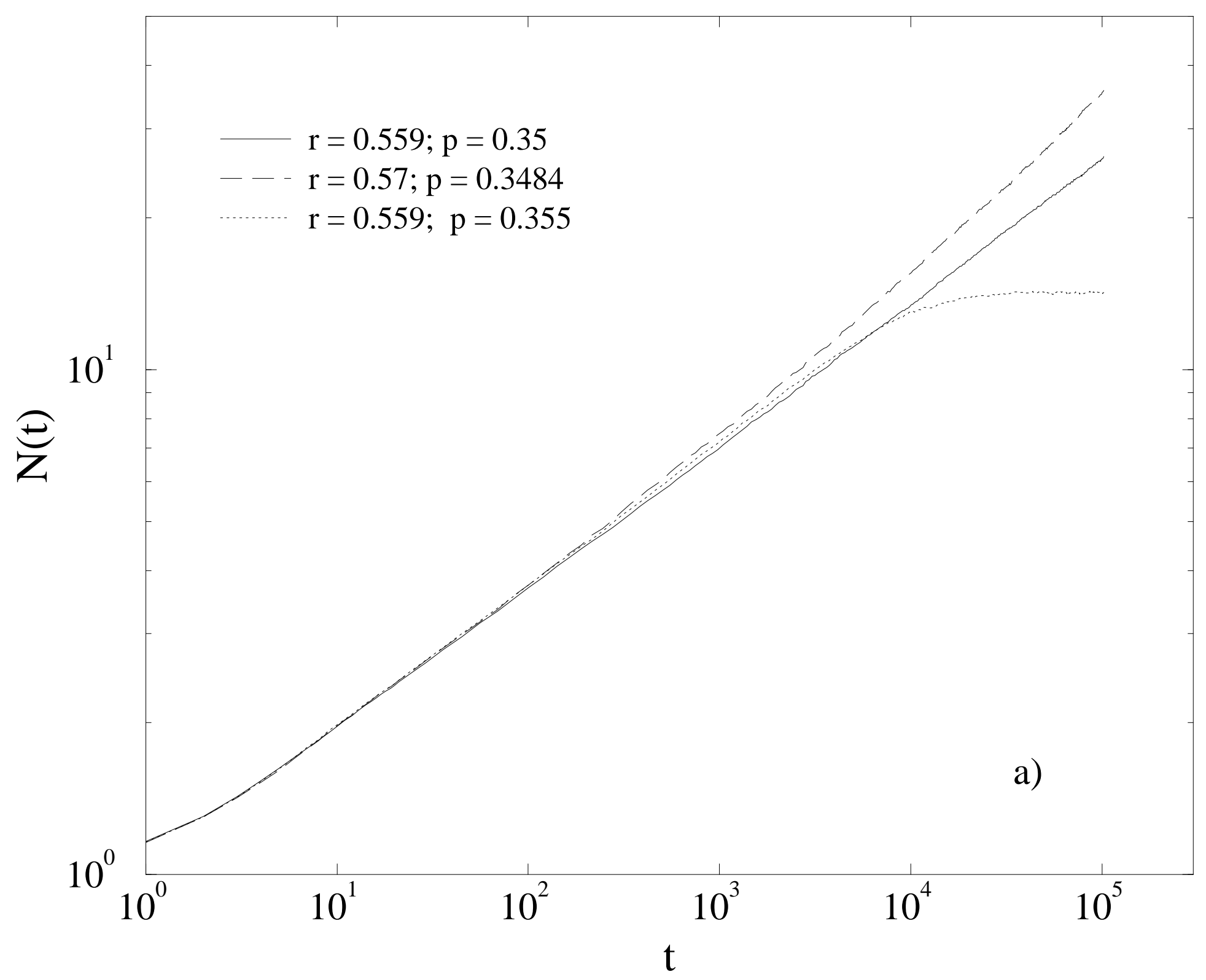




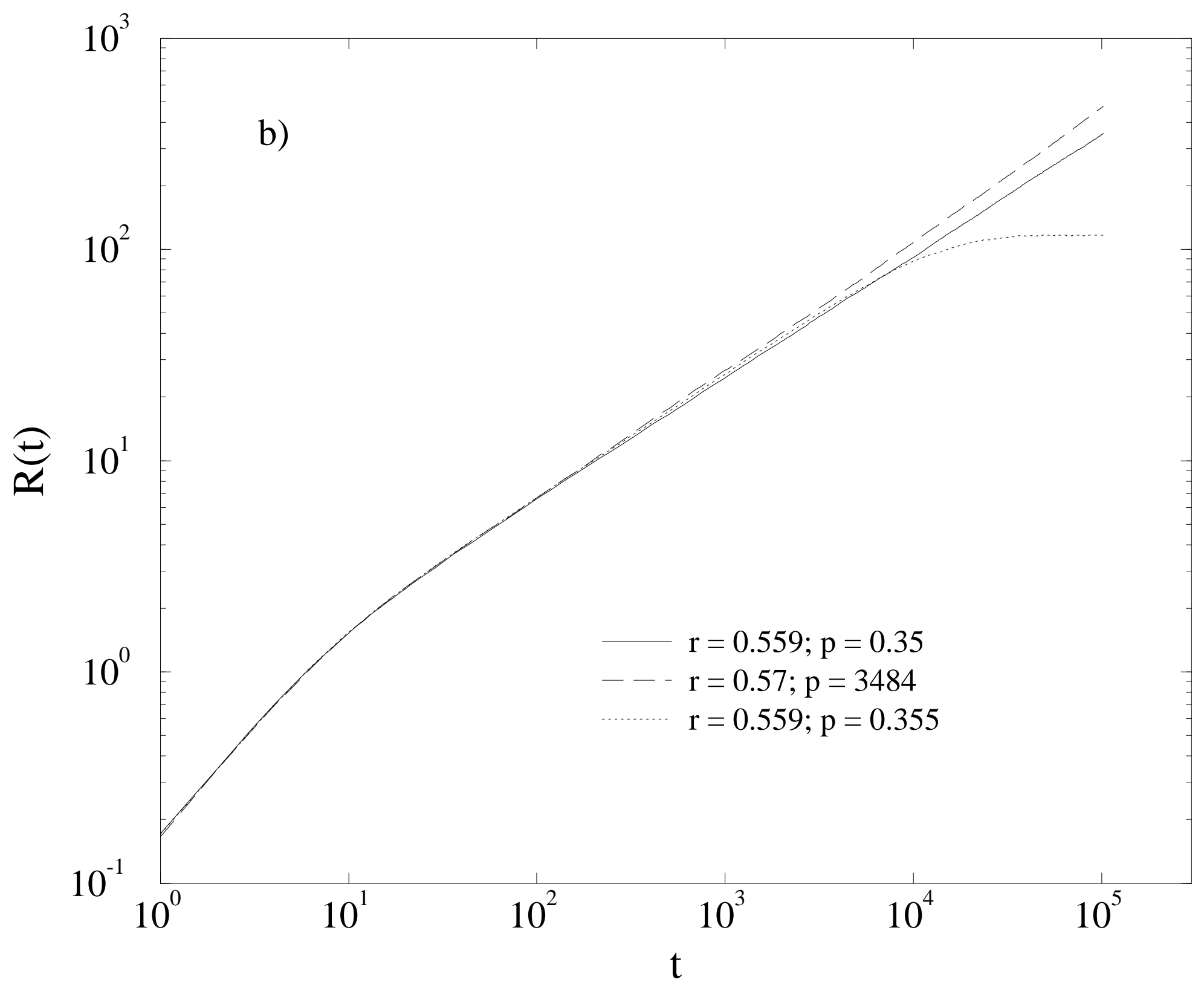




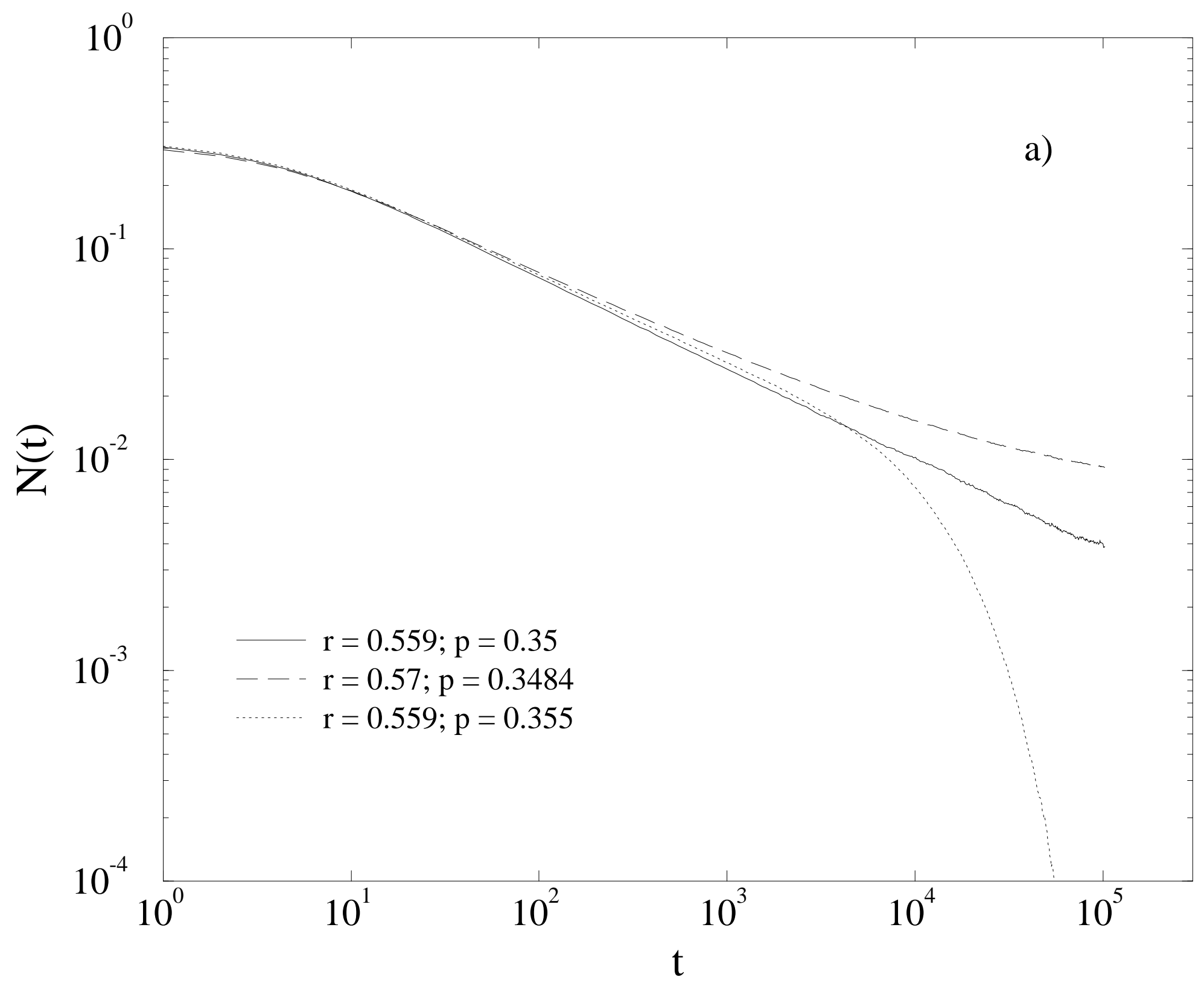




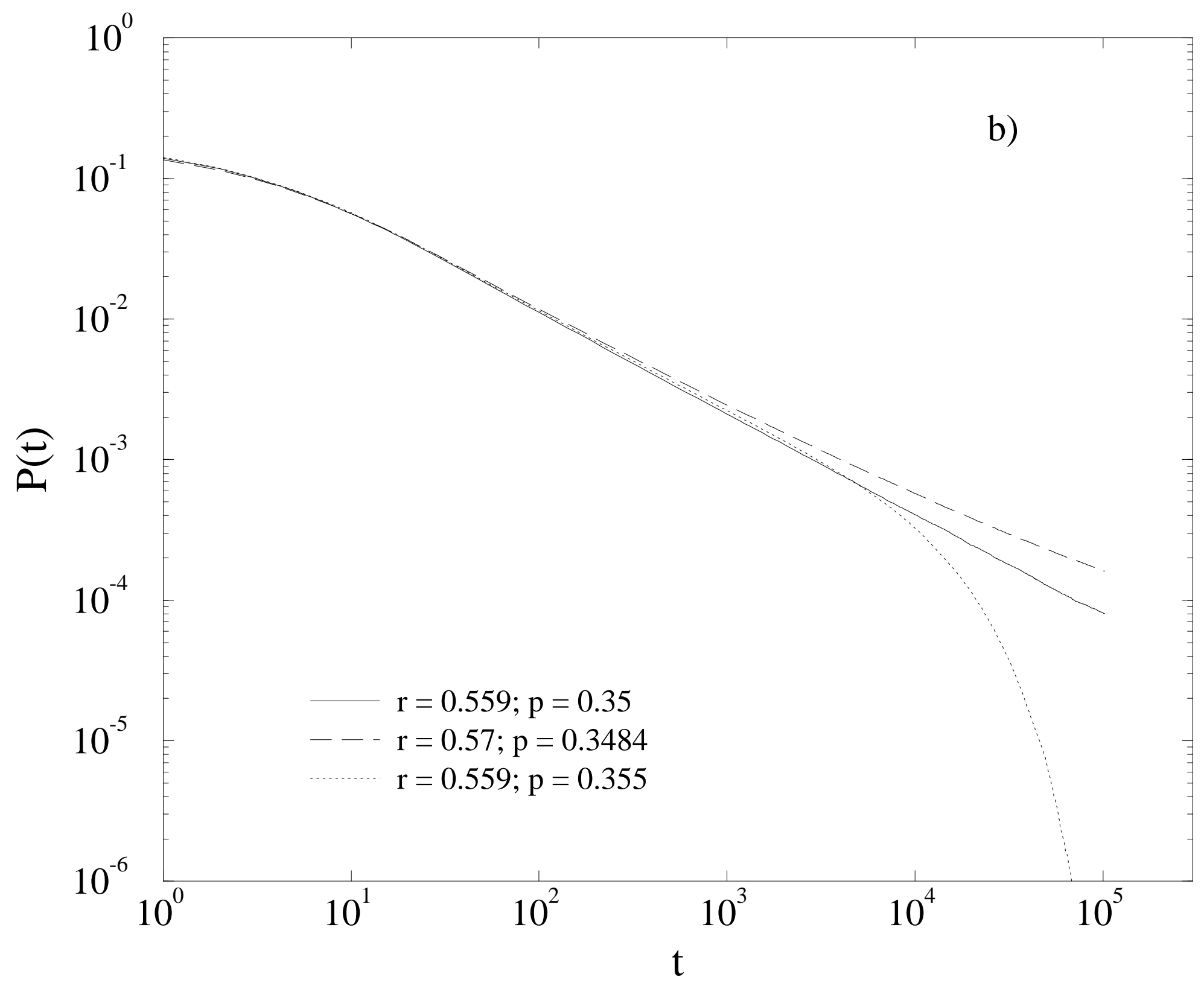




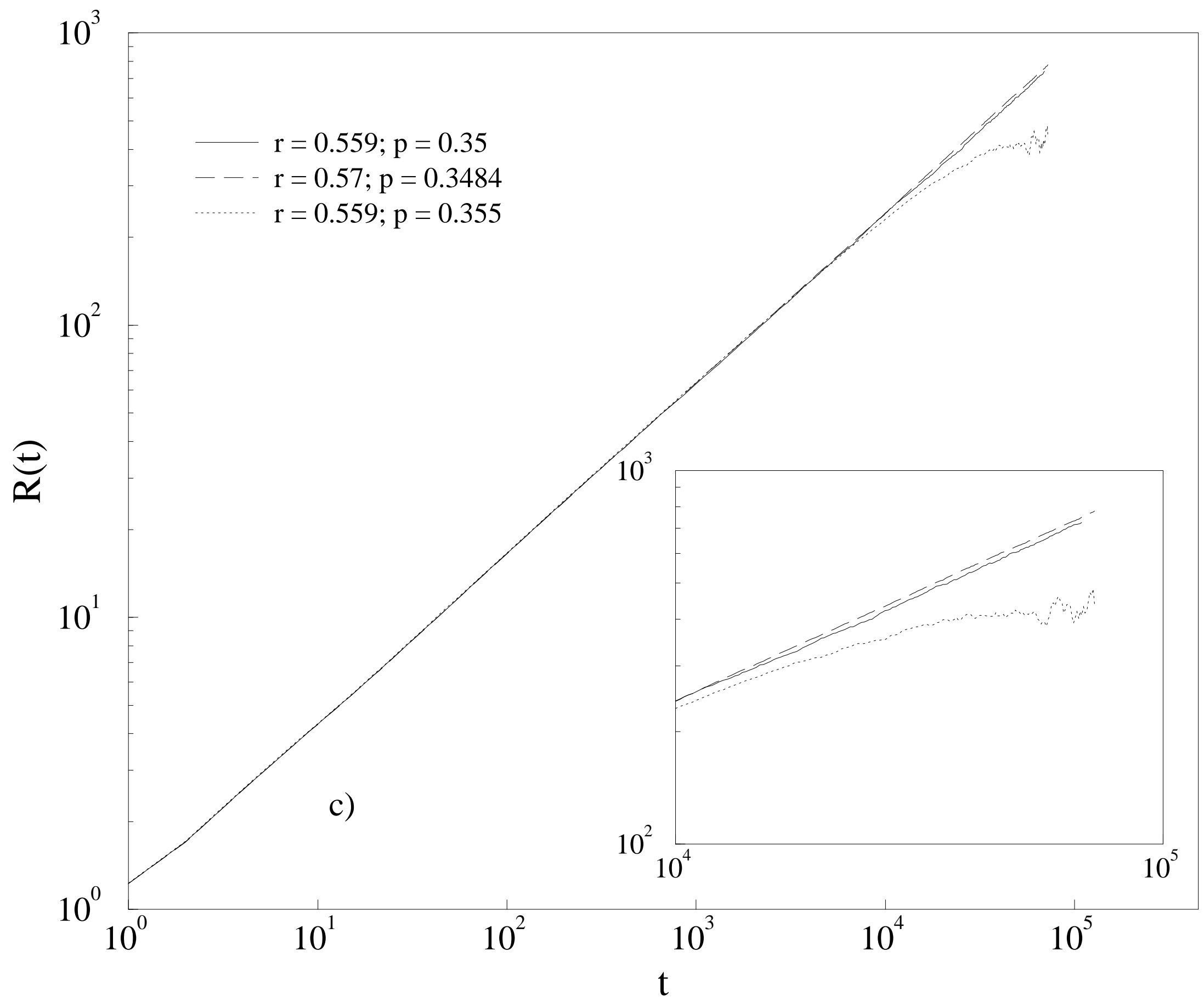

